\title{
Model Reduction and Analysis of Deep Dynamic Stall on a Plunging Airfoil
}

\author{
Arvind T. Mohan ${ }^{1, *}$, Datta V. Gaitonde ${ }^{2, *}$, Miguel R. Visbal ${ }^{3, * *}$
}

\begin{abstract}
The ability to generate massive amounts of high-resolution data, both experimentally and computationally, has led to a surge of interest in mathematical model reduction using modal decomposition algorithms. When applied to complex unsteady flows, different techniques highlight different flow dynamics which are difficult to extract directly from large datasets. A widely used technique is Proper Orthogonal Decomposition (POD) which ranks modes by their relative energy content. A newer method, Dynamic Mode Decomposition (DMD), focuses on rates of growth of different modes, thus retaining temporal information pertinent to dynamic events which dominate highly transient flows. In this work, we employ these techniques, with emphasis on DMD, to analyze flow past an SD7003 airfoil undergoing periodic plunging motion representative of small unmanned vehicles. Snapshots are obtained from a high-fidelity, experimentally validated Large-Eddy Simulation (LES). The stability characteristics of DMD modes show that the flow structure associated with the leading edge vortex (LEV) in dynamic stall is unsta-
\end{abstract}

\footnotetext{
* Mechanical and Aerospace Engineering, The Ohio State University, Columbus, OH

**U.S. Air Force Research Laboratory, Wright-Patterson Air Force Base, Ohio 45433 Email address: mohan. 69 @osu. edu (Arvind T. Mohan)

${ }^{1} \mathrm{PhD}$ Student, Aeronautical and Astronautical Engineering

${ }^{2}$ John Glenn Chair Professor

${ }^{3}$ Principal Research Aerospace Engineer
} 
ble. The influence of DMD modes in local regions of the flow provide insight into which flow frequencies may be targeted by leading edge actuators to have maximum impact in controlling the unstable flow structures inducing stall. Dominant POD modes, each of which can have multiple frequency components, are shown to be comprised primarily of the dominant DMD mode contributions. A practical framework is introduced to identify components of a global flow structure across different velocity components. The method is shown to successfully reproduce the global flow structure. A few dominant DMD modes are used to reconstruct the flow near the leading edge, where rapid changes occur during parts of the plunging cycle and the ability of individual probe to provide insight into global phenomena is assessed. Finally, a stability analysis of each mode is performed to identify flow instabilities near the leading edge.

Keywords: Dynamic Stall, Dynamic Mode Decomposition, Proper Orthogonal Decomposition, LES, Model Reduction

\section{Introduction}

In the recent years, there has been a surge of interest in the aerodynamics of Unmanned Air Vehicles (UAVs) and Micro Air Vehicles (MAVs) due to their demonstrated potential in various civilian and military applications. Such aircraft, especially MAVs, are characterized by complex, unsteady low Reynolds number flows with stability and performance issues due to flow separation and formation of vortices from dynamic stall. Dynamic stall is a highly unsteady phenomenon where airfoils in pitching or plunging motion undergo large scale flow separation, giving rise to unsteady vortices. The attendant non-linear phenomena cause severe variations in the aerodynamic loading profile over the airfoil and loss of lift. A 
few representative early studies of dynamic stall may be found in Refs. [1, 2, 3, 4].

Dynamic stall is characterized by highly unsteady laminar-transitional-turbulent regimes, whose details depend on flight parameters and configurations. Among primary causes of dynamic stall in MAVs are sudden, extreme variations in the effective angle of attack (such as flapping wings and airfoils in rapid maneuvers) and wind gusts which can be quite significant due to their small size. Hence, MAVs are highly susceptible to the inherently detrimental phenomena associated with dynamic stall.

Consequently, there have been numerous efforts in the recent years to prevent stall by various steady or unsteady means. Steady approaches employ static devices such as flaps, riblets etc.[5], in addition to constant blowing jets which add momentum to the flow to effect the desired change [6]. Unsteady techniques include pulsed jets[7] and plasma-based[8, 9, 10] actuators. Pulsed techniques seek to leverage transients in the flow, often through exploitation of natural instabilities. For example, Little et al[10] demonstrate control of static stall with Nano-Second Pulsed Dielectric Barrier Discharges (NS-DBDs) pulsed at Strouhal (St) numbers of $O(1)$. However, the forcing frequency is often based on a trial-and-error approach and empirical data for a given wing-flight regime configuration.

The focus of this work is on dynamic stall, the key phenomena which occurs at short time scales [11]. A knowledge of the transient dynamics can greatly facilitate a better understanding of the flow physics, as well as accelerate the application of high band-width actuators to interfere in instability growth mechanisms. Hence, there is a critical need to have a physics-based approach that elucidates the key dynamics in terms of specific flow features responsible for the initiation of dynamic stall, along with their frequency and stability characteristics. Such an 
analysis allows focus on flow features that must be targeted for control, providing a priori estimates of actuator control characteristics with minimal empiricism.

Mode decomposition and model reduction techniques provide a convenient tool to extract the key modes that describe the flow $[12,13]$. These mathematical tools have seen increasing popularity in recent literature for their ability to isolate key features from large datasets, allowing for more efficent data analysis. Mathematical model reduction involves decomposing high-dimensional data, such as CFD simulations or PIV from experiments, into constituent low-dimensional components known as modes which can then be used to study the aspects of the flow which are most important from an application standpoint. Additionally, reduced order models of the flow-field can also be built from these modes which are highly useful to study complex turbulent flows on a much more tractable scale.

In this paper, we use Dynamic Mode Decomposition (DMD) in conjunction with a validated Large-Eddy Simulation (LES) to analyze some of the global features of dynamic stall. The advantage of DMD is its capability to highlight features with varying growth or decay rates [12]. Some results are also presented with POD to highlight its complementary nature. The specific case considered is an SD 7003 airfoil undergoing a periodic plunging motion in a manner that closely replicates actual MAV flight conditions. Details on how the dataset is generated have been described in Ref. [11]. The computation has been verified with PIV results from two independent experiments at AFRL and the University of Michigan. We perform analyses with the goal of identifying flow frequencies and their spatial structures using DMD since traditional methods like FFT, STFT etc. cannot isolate entire spatial domains at a single frequency. Furthermore, the behavior and stability characteristics of these flow frequencies have been studied, 
to understand the physics behind stall initiation and propagation, and its qualitative and quantitative features have been described in detail. The motivation for this analysis stems from the aforementioned fact that successful identification and characterization of the flow structures causing dynamic stall could be eventually used to predict suitable actuation parameters for leading edge active control. In order to explore this further, local signatures of the spatially global DMD modes near the leading edge have been investigated. Finally, we attempt to establish a relationship between the POD and DMD modes as understanding this relationship would be very beneficial since practitioners of these tools can get a more unified insight into datasets of engineering interest.

Proper Orthogonal Decomposition (POD) has been widely used among the turbulence community after it was popularized by Lumley et. al [14]. POD ranks the modes by the decreasing order of energy, and is the most optimal energy basis for a given dataset. The energies of these modes are found from their singular values. Physically, the mode ranked the highest i.e. Mode 1, having the greatest energy represents the mean flow. POD has been used to study numerous practical cases $[15,16,17]$ in literature, with varying degrees of success. POD was also used to study dynamic stall by Mulleners et. al [18]

The primary tool employed in this work is Dynamic Mode Decomposition (DMD). The approach was introduced relatively recently $[12,19,13]$ but is rapidly gaining popularity as a complement to POD. Unlike POD which targets energy content, DMD ranks modes by their most dynamically changing features[19] even if they have low energy content. Since it retains temporal information, it is attractive for highly unstable and transient flows such as the plunging airfoil as well as for noisy, complex turbulent flows. The DMD algorithm is an implementation and 
approximation of the Koopman decomposition technique for nonlinear dynamical systems[13].

Briefly, DMD computes a finite-dimensional approximation of the infinitedimensional Koopman operator using a sequence of vector valued measured data from simulation or experiment[20]. DMD effectively isolates the most dynamically significant modes containing flow structures oscillating at a single frequency. Additionally, the modes are ranked by the amplitude/energy of these flow frequencies, but the energy in these modes are not optimal as in POD. Apart from decomposing the flow field into frequencies, the complex eigenvalues of the approximate Koopman operator in DMD reflects the stability characteristics of the mode. Algorithms to compute DMD may be found in Refs. [21, 22]. There has also been focus on developing DMD based model reduction algorithms such as Sparsity-promoting DMD [23],Optimal mode decomposition [24],Optimized DMD [20] and Chronos-Koopman Spectral Decomposition [25]. In applications, DMD has been shown to work well for analyzing highly transient cases like flow in a cavity[19, 26], wakes behind bodies [12, 27, 22, 28, 29], detonation waves [30] and several jet cases[13, 31, 12, 19, 32, 33].

The present work uses DMD to analyze a complex flow problem of a wing undergoing deep dynamic stall. Although there is widespread interest in applying DMD to practical problems, literature on analysis of airfoils is scarce. DMD and POD were used to analyze dynamic stall of a pitching airfoil in 2D [34], particularly the effect of neglecting the boundary layer in PIV measurements and reduced order models using dominant DMD modes were built. Ref.[35] contains a very brief demonstration of DMD to study the effect of flaps in a 2D NACA0015 airfoil. However, very little work has been done in using DMD to obtain 
physically-relevant insight into airfoil cases of practical interest.

The conditions in the present work replicate realistic conditions for which a high-fidelity, validated 3-D dataset is available from which spanwise averaged unsteady snapshots are obtained over two cycles. The focus is on isolating flow frequencies associated with dynamic stall and building a reduced order model of the flow using those frequencies to better understand the physics of the phenomenon. As mentioned earlier, the eventual goal is to use the insight gained from this analysis to propose effective control strategies using plasma actuators. We first summarize the LES database (Section 2), then the DMD methodology (Section 2.3). The DMD and POD modes are described in Sections 3.1 and 3.2. Their relationship is delineated in the context of flow structure identification in Section 3.3. The use of DMD to identify modal content in localized regions is discussed in Section 3.4. The ability of DMD to reconstruct the solution from a small subset of modes is analyzed in Section 3.5. Most of the results are presented using the streamwise component of velocity to perform the decompositions. In Section 3.6, the consequences of using the vertical component of velocity is examined. Finally, the stability characteristics are analyzed in Section 3.7.

\section{Large-Eddy Simulations used to obtain Snapshots}

The dynamic stall dataset employed for the analysis has been described in Visbal[11]. A summary is now provided for reference. An SD7003 airfoil with a chord length $c=0.1$ has been chosen for computations since it was originally designed for low Reynolds number applications. It has a maximum thickness of $8.5 \%$ and maximum camber of $1.45 \%$ at $x / c=0.35$. The airfoil has a spanwise length $s$ of $s / c=0.4$. The chord Reynolds number $R e_{c}$ has been fixed at $6 \times 10^{4}$, 


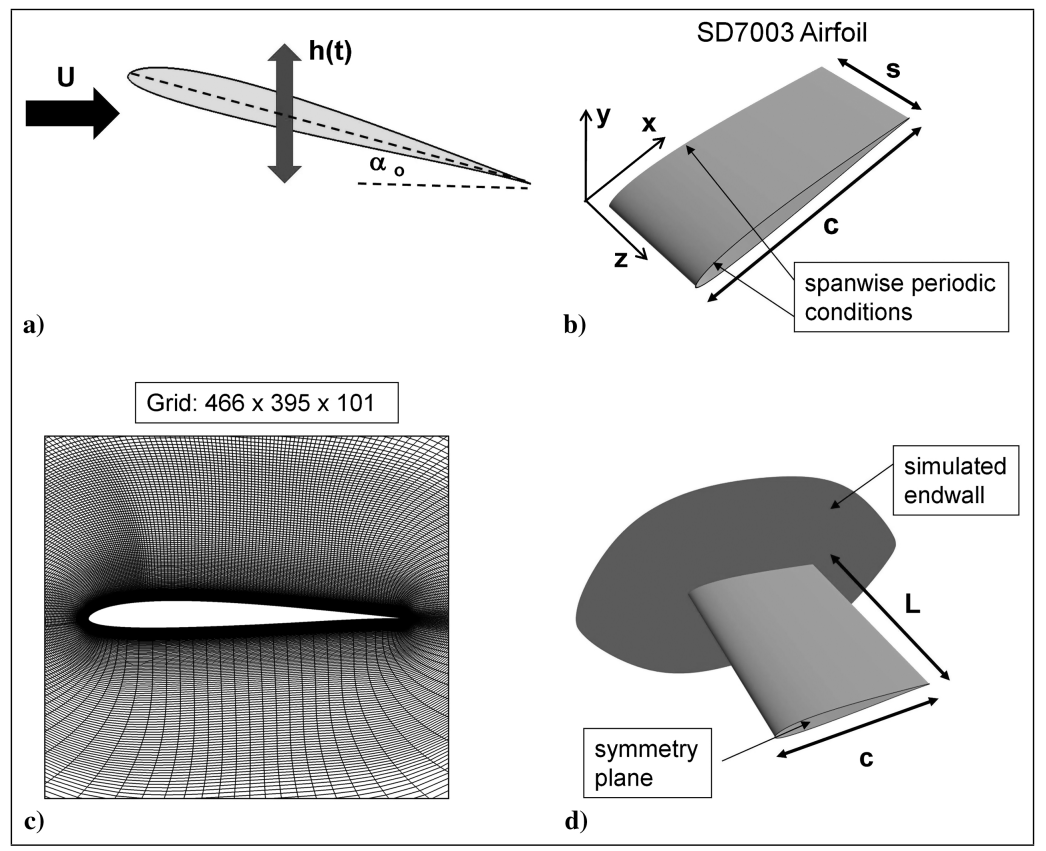

Figure 1: Airfoil configuration and computational mesh

for which experimental results were available for validation purposes.

\subsection{Numerical Scheme}

An extensive grid resolution study, including mesh density as well as spanwise domain extent, has been presented in Ref. [11]. Based on these, results are analyzed on an O-mesh of $466 \times 395 \times 101$. Figure 1 shows the computational mesh and the domain used in the simulation. A grid resolution study was performed in Visbal[11] with four grid sizes - a) $315 \times 151 \times 51$ b) $390 \times 242 \times 51$ c) $466 \times 395 \times 51$ and d) $466 \times 395 \times 101$. Mesh (d) was found to be most suitable for the study and was thus used for the simulation. The grid points near the airfoil are highly concentrated near the surface to capture the boundary layer behaviour during the dynamic stall process. 
The flow field snapshots are generated by spanwise averaging instantaneous results obtained with an implicit large-eddy simulation (ILES) approach. The spatial derivatives are evaluated with a sixth-order Pade-type scheme, combined with a low pass filter which provides regularization in turbulent flow regions. This method is found to be particularly attractive for the present application, wherein a seamless approach capable of handling mixed laminar, transitional, and turbulent flows is needed, and also does not require the use of a dedicated subgrid scale model for LES.

For the boundary conditions, a no-slip adiabatic condition is employed in conjunction with a zero normal pressure gradient along the airfoil surface. The wall velocity components $\left(u_{s}, v_{s}, w_{s}\right)$ are specified based on the plunging motion:

$$
\begin{aligned}
& u_{s}=-\dot{h} \sin \alpha_{0}, \\
& v_{s}=\dot{h} \cos \alpha_{0}, \\
& w_{s}=0
\end{aligned}
$$

where,

$$
\begin{aligned}
& h(t)=h_{0} \sin [2 k F(t) t] \quad \text { where, } \\
& F(t)=1-e^{-a t}, \quad a=4.6 / t_{0}, \quad t_{0}=0.25
\end{aligned}
$$

where $\alpha=8^{\circ}$ is the static mean angle of attack, $h_{0}=0.5$ the nondimensional plunging amplitude normalized with the chord length $c, k=\frac{\pi f c}{U_{\infty}}$ is the reduced frequency, and $F(t)$ is a ramping function that smoothly transitions the airfoil from a stationary position to the desired periodic motion. These motion parameters correspond to an induced angle-of-attack range of $-6^{\circ} \leq \alpha_{\text {eff }} \leq 22^{\circ}$. The maximum $\alpha_{\text {eff }}$ promotes unsteady leading-edge separation, leading to deep dy- 
namic stall. The Strouhal number in this simulation is defined as $S t=\frac{2 h_{0} f}{U_{\infty}}$, which for the plunging motion is $S t=0.0793$.

\subsection{Description of Dynamic Stall Behavior}

For subsequent reference, this section summarizes the analysis of Ref. [11]. The primary aspects of the flow in a typical cycle are shown with respect to the phase of the plunging cycle in Fig. 2, with $\theta=0^{\circ}, 90^{\circ}, 180^{\circ}$ and $\theta=270^{\circ}$ corresponding to positions of maximum upward displacement, maximum downward velocity, maximum downward displacement and maximum upward velocity respectively. The flow over the airfoil becomes laminar during its upward motion, with shedding of small near-periodic trailing edge vortices. However, after $\theta=20^{\circ}$, (when the descent phase begins) small reversed flow regions form near the trailing edge which travel forward to the leading edge. These structures grow rapidly in size as the airfoil descends. As such, the entire process of transition from laminar to turbulent boundary layer is very rapid. The shear layer at the leading edge interacts with the airfoil surface and essentially ejects vortices from its surface. This quickly leads to the complete breakdown of the shear layer near the leading edge - thus forming the leading edge vortex (LEV). Further, these ejected vortices impinge on the boundary layer downstream resulting in a large number of turbulent small scale structures. By $\theta=120^{\circ}$, the flow is completely separated with the LEV being the dominant structure in the flow, and a large wake is observed. At the same time, a small trailing edge vortex is formed which grows as the airfoil descends. Towards the end of the plunge $\theta=180^{\circ}$ (the point of maximum downward displacement) this vortex breaks down into several structures. At this point the flow over the airfoil is completely turbulent and separated without any obvious large coherent structures. After this, the upward motion of 

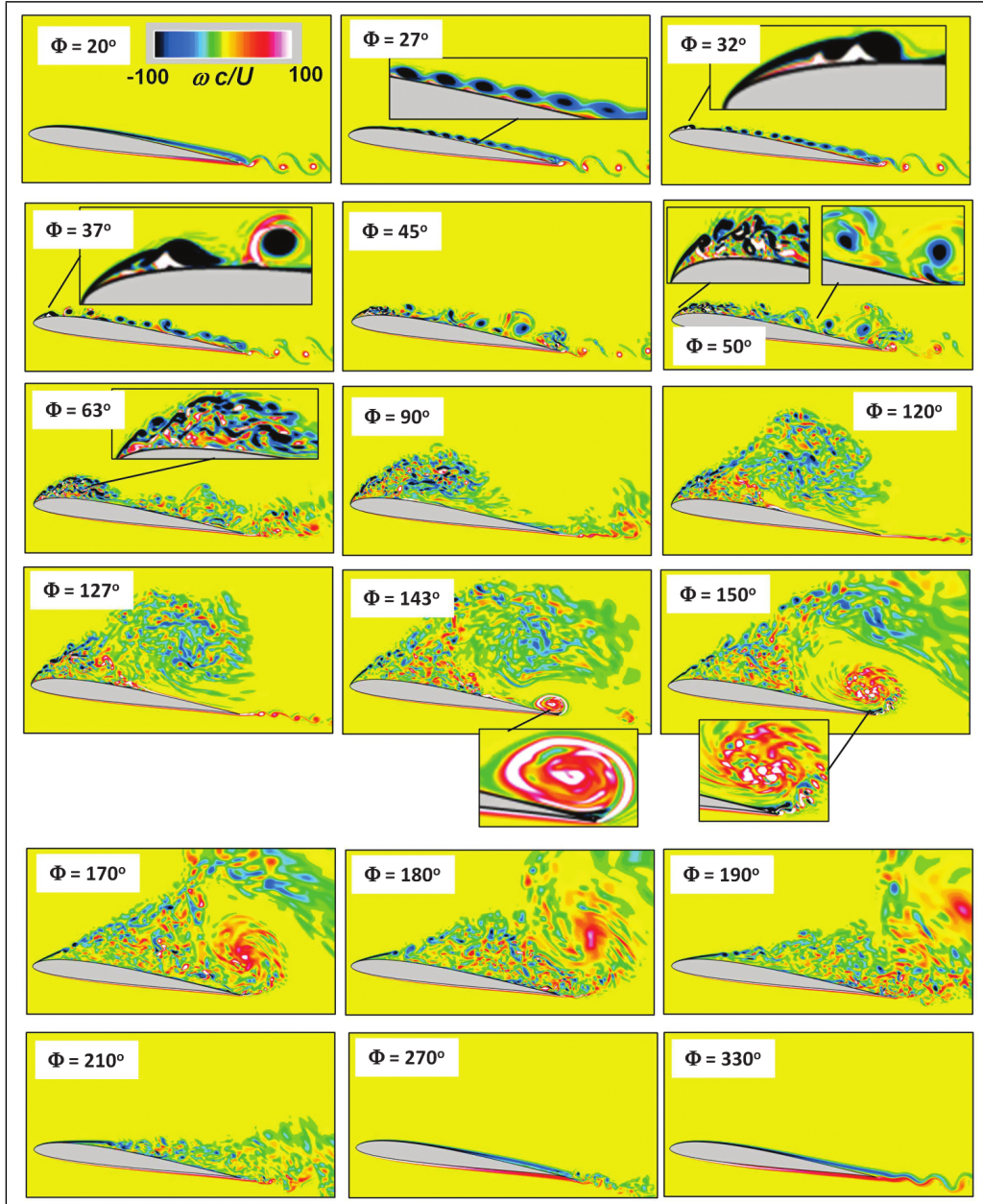

Figure 2: Computed instantaneous spanwise vorticity at selected phases of plunging cycle. From [11] 
the airfoil begins and the flow is quickly stabilized and reattaches to the surface, leading to the laminar boundary layer with periodic trailing edge wakes as seen at $\theta=30^{\circ}$. The cycle then repeats.

\subsection{Dynamic Mode Decomposition}

The DMD algorithm [12] used in this work considers a series of "snapshots" of the flow past the airfoil. Parameters such as sampling frequency and preprocessing of the LES results are presented in Section 3. These snapshots, $\mathbf{v}_{i}$ can be arranged in a matrix $\mathbf{V}_{\mathbf{1}}^{\mathbf{N}}$ and two lagged matrices $\mathbf{V}_{\mathbf{1}}^{\mathbf{N}-\mathbf{1}}$ and $\mathbf{V}_{\mathbf{2}}^{\mathbf{N}}$ are constructed:

$$
\begin{aligned}
\mathbf{V}_{\mathbf{1}}^{\mathbf{N}} & =\left\{\mathbf{v}_{\mathbf{1}}, \mathbf{v}_{\mathbf{2}}, \mathbf{v}_{\mathbf{3}} \ldots . \mathbf{v}_{\mathbf{N}}\right\} \\
\mathbf{V}_{\mathbf{1}}^{\mathbf{N}-\mathbf{1}} & =\left\{\mathbf{v}_{\mathbf{1}}, \mathbf{v}_{\mathbf{2}}, \mathbf{v}_{\mathbf{3}} \ldots \mathbf{v}_{\mathbf{N}-\mathbf{1}}\right\} \\
\mathbf{V}_{\mathbf{2}}^{\mathbf{N}} & =\left\{\mathbf{v}_{\mathbf{2}}, \mathbf{v}_{\mathbf{3}}, \mathbf{v}_{\mathbf{4}} \ldots \mathbf{v}_{\mathbf{N}}\right\}
\end{aligned}
$$

A linear dynamical propagator, $\mathbf{A}$, is defined as an approximation to the nonlinear mapping propagator. This operator governs the evolution of the state of the system from one time instant to the next.

$$
\mathbf{v}_{\mathbf{i}+\mathbf{1}}=\mathbf{A} \mathbf{v}_{\mathbf{i}}
$$

The linear system can be represented as

$$
\mathrm{AV}_{1}^{\mathrm{N}-1}=\mathrm{V}_{2}^{\mathrm{N}}
$$

Employing a Krylov sequence in terms of $\mathbf{A}$ and $\mathbf{v}_{\mathbf{1}}$,

$$
\mathbf{V}_{\mathbf{1}}^{\mathbf{N}}=\left\{\mathbf{v}_{\mathbf{1}}, \mathbf{A} \mathbf{v}_{\mathbf{1}}, \mathbf{A}^{2} \mathbf{v}_{\mathbf{1}}, \ldots, \mathbf{A}^{N-1} \mathbf{v}_{\mathbf{1}}\right\}
$$


the final snapshot can be expressed as a linear combination of the preceding snapshots. This assumption is made for a sufficiently large number of snapshots, until the vectors in the sequence become linearly dependent

$$
\mathbf{v}_{\mathbf{n}}=a_{1} \mathbf{v}_{\mathbf{1}}+a_{2} \mathbf{v}_{\mathbf{2}}+\ldots .+a_{N-1} \mathbf{v}_{\mathbf{N}-\mathbf{1}}+\mathbf{r}
$$

where $\mathbf{r}$ denotes the residual, for which a lower value indicates better convergence. Writing $\mathbf{a}^{T}$ as $\mathbf{a}^{T}=\left\{a_{1}, a_{2}, a_{3}, \ldots \ldots, a_{N-1}\right\}$,

$$
\mathbf{v}_{\mathbf{n}}=\mathbf{V}_{\mathbf{1}}^{\mathbf{N}-\mathbf{1}} \mathbf{a}+\mathbf{r}
$$

An approximation for the A matrix is needed since there is no a priori knowledge of it. Thus, a companion matrix $\mathbf{S}$ is built as the required approximation by expressing the final snapshot as a linear combination of the previous snapshots.

$$
\begin{gathered}
\mathbf{A}\left\{\mathbf{v}_{\mathbf{1}}, \mathbf{v}_{\mathbf{2}}, \mathbf{v}_{\mathbf{3}} \ldots \mathbf{v}_{\mathbf{N}-\mathbf{1}}\right\}=\left\{\mathbf{v}_{\mathbf{2}}, \mathbf{v}_{\mathbf{3}}, \mathbf{v}_{\mathbf{4}} \ldots \mathbf{v}_{\mathbf{N}}\right\} \\
\mathbf{A}\left\{\mathbf{v}_{\mathbf{1}}, \mathbf{v}_{\mathbf{2}}, \mathbf{v}_{\mathbf{3}} \ldots \mathbf{v}_{\mathbf{N}-\mathbf{1}}\right\}=\left\{\mathbf{v}_{\mathbf{2}}, \mathbf{v}_{\mathbf{3}}, \mathbf{v}_{\mathbf{4}} \ldots \mathbf{V}_{\mathbf{1}}^{\mathbf{N}-\mathbf{1}} \mathbf{a}\right\}+\mathbf{r e}_{N-1}^{T} \\
\mathbf{A V}_{\mathbf{1}}^{\mathbf{N}-\mathbf{1}} \equiv \mathbf{V}_{\mathbf{1}}^{\mathbf{N}-\mathbf{1}} \mathbf{S}++\mathbf{r e}_{N-1}^{T} \\
\mathbf{V}_{\mathbf{2}}^{\mathbf{N}} \equiv \mathbf{V}_{\mathbf{1}}^{\mathbf{N}-\mathbf{1}} \mathbf{S}+\mathbf{r e}_{N-1}^{T}
\end{gathered}
$$

Finally, $\mathbf{S}$ is of the form,

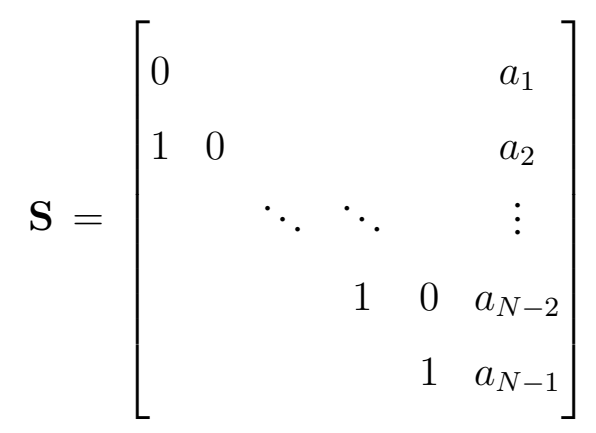


To be able to compute the S matrix, a Singular Value Decomposition (SVD) of $\mathbf{V}_{1}^{N-1}$ is used.

$$
\begin{aligned}
S V D\left(\mathbf{V}_{1}^{N-1}\right) & =\mathbf{U} \boldsymbol{\Sigma} \mathbf{W}^{H} \\
\mathbf{V}_{2}^{N} & =\mathbf{U} \boldsymbol{\Sigma} \mathbf{W}^{H} \mathbf{S}
\end{aligned}
$$

Finally, the $\tilde{\mathbf{S}}$ matrix can be obtained by projecting $\mathbf{A}$ on the subspace of $\mathbf{U}$.

$$
\tilde{\mathbf{S}} \equiv \mathbf{U}^{H} \mathbf{V}_{2}^{N} \mathbf{W} \boldsymbol{\Sigma}^{-1}
$$

$\tilde{\mathbf{S}}$ is the approximation for $\mathbf{A}$ which we sought, which is obtained by projecting A on the $\mathrm{U}$, which contain the POD basis [12]. The DMD modes are finally computed with an eigenvalue decomposition of the $\tilde{\mathbf{S}}$ matrix, resulting in eigenvectors $\mathbf{y}_{i}$ and eigenvalues $\mu_{i}$ such that

$$
\tilde{\mathbf{S}} \mathbf{y}_{i}=\mu_{i} \mathbf{y}_{i}
$$

The DMD modes $\Phi$ are then given by

$$
\Phi_{i}=\mathbf{U y}_{i}
$$

where $\mathbf{U}$ is the right singular vector of $\mathbf{V}_{\mathbf{1}}^{\mathbf{N}-\mathbf{1}}$ and $\mathbf{y}_{i}$ are the eigenvectors of $\tilde{\mathbf{S}}$. The approximate eigenvalues $\mu$ (Ritz values) obtained from DMD are expressed by,

$$
\lambda=\frac{\log (\mu)}{\Delta t}
$$

where $\Delta t$ is the time separation between subsequent snapshots. These eigenvalues can be used to study the stability characteristics of the modes. Positive $\Re(\lambda)$ and negative $\Re(\lambda)$ values denote unstable and stable modes respectively. A neutrally stable mode has a zero real part. A special case is the mean flow, which has a zero 
eigenvalue, indicating that it is time-invariant (zero frequency). The eigenvalues usually arise as complex conjugate pairs, thus leading to complex conjugate eigenvectors (modes). This is a consequence of using a real valued data matrix. These conjugate modes have the same stability characteristics and frequencies but a different sign. Since the negative frequencies are neglected, each mode pair is identified by only the mode with the positive-valued frequency.

The more commonly used POD modes are computed using equation shown below. The method of snapshots [14] is also a viable alternative to compute the POD modes.

$$
S V D\left(\mathbf{V}_{\mathbf{1}}^{\mathbf{N}-\mathbf{1}}\right)=\mathbf{U} \Sigma \mathbf{W}^{H}
$$

where $\mathbf{U}$ contains the spatial structures, and $\Sigma$ is the diagonal matrix of eigenvalues which ranks the spatial structures based on their energy content. $\mathbf{W}$ has the temporal information of the POD modes.

\section{Results}

A region surrounding the airfoil has been used for the analysis, as shown in Fig. 4 which consists of a slice taken around the suction surface of the airfoil, where most of the phenomena of interest are present. Since the focus of this paper is on the larger scale structures, we consider two plunging cycles. A total of 720 3D unsteady snapshots were generated from high-fidelity LES, and each of these were averaged in the spanwise direction, along the z-axis (The airfoil cross section lies in the XY plane) to get 720 2D unsteady snapshots. These snapshots were sampled in intervals of 280 time-steps, where the time-step refers

to the LES $\Delta t$ of $1.25 \times 10^{-4}$. The $\Delta t$ is non-dimensionalized by expressing it as $\Delta t U_{\infty} / c$, where $c$ is the chord length and $U_{\infty}$ is the free-stream velocity 
based on the a low free-stream Mach number of $M_{\infty}=0.1$ used for the simulation. Therefore, the sampling rate of the snapshots for DMD input corresponds to $\left(1.25 \times 10^{-4}\right) * 280=3.5 \times 10^{-2}$. This sampling rate captures the primary structures of interest in the flow, as confirmed by the subsequent results. However, small scale highly-fluctuating structures in the shear layer emanating cannot be captured completely with the current sampling rate. Those will be the focus of a future effort. Hence, all the studies in this work were performed with the chosen sampling rate. As mentioned in Section 2.3, the DMD modes have been ranked by their normalized energy i.e., normalized by the mode with highest energy. For statistically stationary snapshots, the highest energy is present in the stationary mode, representing the mean flow spatial structure. Higher DMD modes appear as complex conjugates and therefore each complex mode pair can be conveniently referred to using just one of the modes for brevity, e.g. mode pair 2 and 3 are referred to as mode 3. Most of the analysis has been done using the streamwise (U) velocity component of the total flow, unless otherwise mentioned. This is because the axial velocity component is the most significant factor in the effective angle-of-attack of the wing, and hence is a favorable candidate to analyse the behavior of dynamic stall. Nevertheless, to completely identify the stall inducing flow structures an analysis of the $\mathrm{V}$-velocity component has also been shown in Section 3.6. The DMD and POD modes resulting from the algorithm have unit norm and hence the velocity contours are shown without the number scales, since they are visualized only for qualitative purposes.

\subsection{DMD Modes}

As noted earlier, the real part of the eigenvalues of the DMD modes provide information about the stability of the associated flow structures. The imaginary 
part of the complex eigenvalue on the other hand, $\Im(\lambda) / 2 \pi$, denotes the frequency of the corresponding mode. Since the real part of the eigenvalue describes the stability of the mode, it can also be used as a criterion to rank the modes from the most unstable to the least unstable mode. However, in the present case, the most unstable mode represents the highly transient, small scale flow structures in the far wake region or the trailing edge vortex shedding. These are not useful to study the process of dynamic stall by LEV formation. Thus, an energy based ranking, which orders the modes by the net contribution of that flow structure to the total flow-field, is more suitable from an application perspective and is adopted here. It is reiterated that the energy in the DMD modes is not the same as the energy in the POD modes. The energy in the POD dominant modes are optimal, given by their eigenvalues, while the DMD modal energy corresponds to the Euclidean norm of the scaled modes.

The dominant $1^{\text {st }}$ mode from POD and DMD are shown in Fig.3a and Fig.3b respectively. As this mode contains the highest norm energy and has zero DMD eigenvalue $\lambda_{i}$, it is the "stationary mode" indicating that the flow structure is neither growing nor decaying with time, and represents the mean flow. The mean flow profile captured by DMD is very similar in comparison to the time-averaged mean in Fig. 4, thus serving as a simple validation of the statistical stationarity of the dataset. If the flow snapshots are of a case that is not statistically converged, the DMD stationary mode shows some growing/decaying behavior with a nonzero frequency component. This result thus serves as a confirmation that number of snapshots is sufficient for the present analyses.

DMD Modes higher than the first are used for further analysis and identification of flow structures associated with dynamic stall. The spatial structures of 


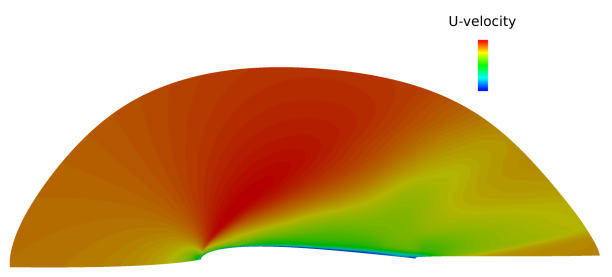

(a) POD Mode 1 (Mean)

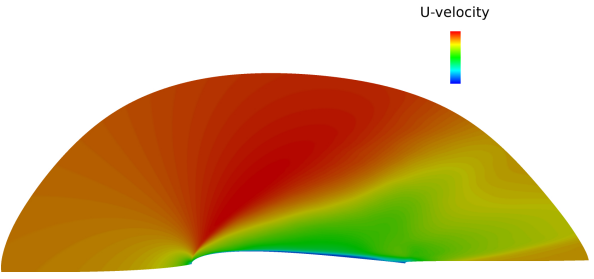

(b) DMD Stationary Mode

Figure 3: Comparison of Mean flow representation by POD and DMD modes

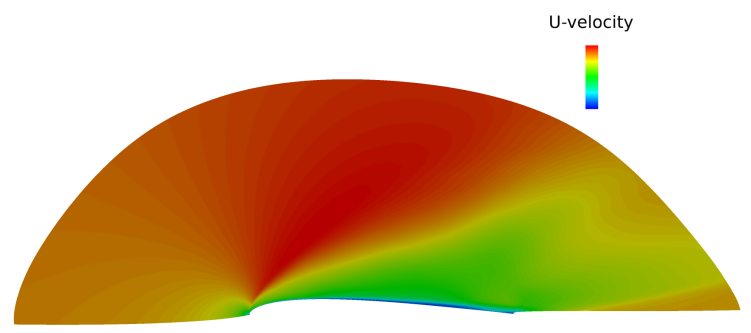

Figure 4: Time Averaged Mean flow

the dominant 11 modes, barring the mean mode 1 are shown in Fig. 5, with only the positive frequency mode representing each mode pair since they both have the same structure. Mode 3 is the most dominant DMD mode apart from the mean, with $S t=0.0793$. This frequency is in fact that of the imposed plunging motion of the airfoil, as mentioned in Section 2. The flow structure in Fig. 5a shows a large recirculation region over the airfoil chord, popularly known in literature as the leading edge vortex (LEV), originating through separation from the leading edge. The structure forms during the downward motion of the airfoil but is suppressed during the upward stroke.

Table 1 shows the dominant DMD modes and their associated frequency values. Modes 5, 7, 9 and 11 are harmonics of the dominant dynamic mode 3. Since the DMD modes are ordered by the highest amplitude, the frequency distribu- 


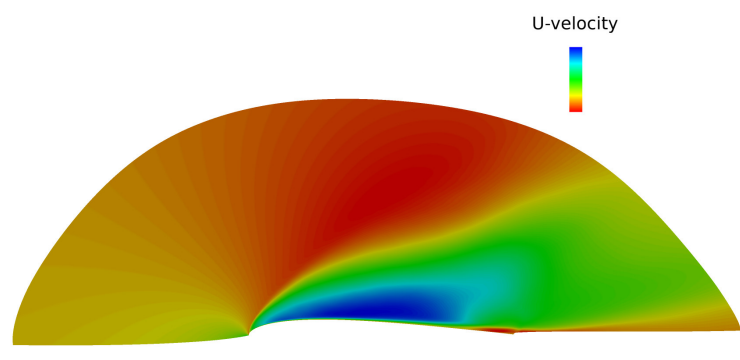

(a) Dominant Dynamic Mode 3

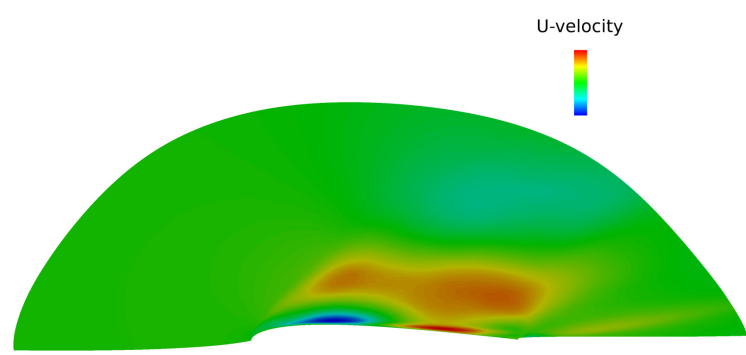

(c) $2^{\text {nd }}$ Harmonic Mode 7

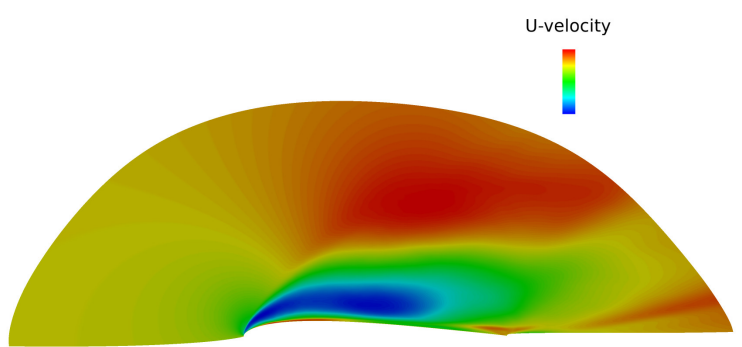

(b) $1^{\text {st }}$ Harmonic Mode 5

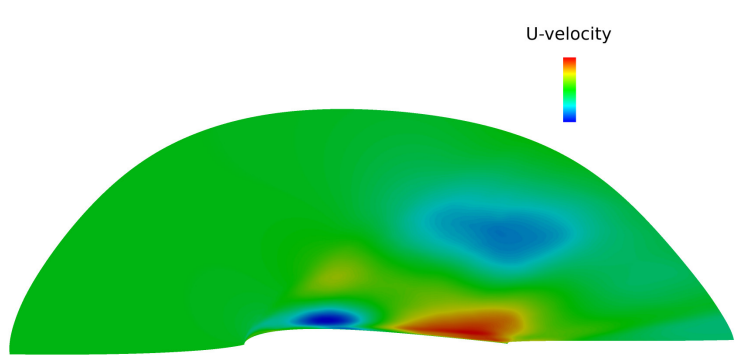

(d) $3^{\text {rd }}$ Harmonic Mode 9

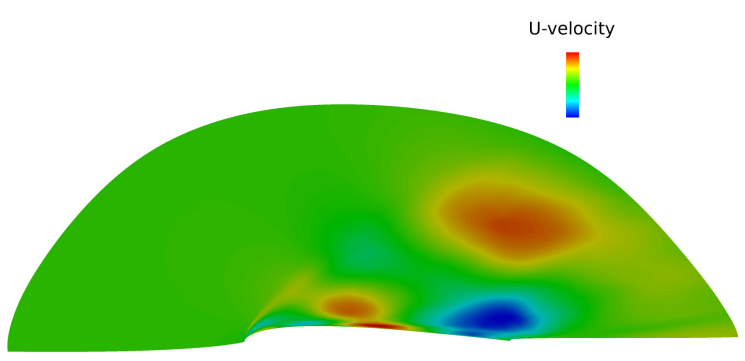

(e) $4^{\text {th }}$ Harmonic Mode 11

Figure 5: Dominant Dynamic DMD modes 


\begin{tabular}{|c|c|}
\hline Mode No. & Frequency $(\Im(\lambda) / 2 \pi)$ \\
\hline 1 & 0 \\
\hline 3 & 0.0793 \\
\hline 5 & 0.1584 \\
\hline 7 & 0.2374 \\
\hline 9 & 0.3160 \\
\hline 11 & 0.3960 \\
\hline
\end{tabular}

Table 1: Frequencies of dominant DMD modes

tion among DMD modes can be studied by plotting DMD frequency with their respective amplitudes.

Figure 6 shows the plot of DMD modes ordered by their amplitudes with their corresponding frequencies. As expected, the DMD mode 1 representing the mean has zero frequency and highest amplitude, as shown by the plot at y-axis of 1. It can be seen that the mode amplitudes are significantly high for frequencies upto $\mathrm{St} \approx 1.5$, beyond which the amplitudes start becoming asymptotically close to a minimum value. The dynamic modes having low frequencies tend to have high amplitudes, although this may not be the case for DMD modes representing very small flow structures since they can have a low amplitude and low frequency as well. Indeed, in the range of St No. from 0 - 1.5 one can see several modes with both low and high amplitudes. It is seen that the high amplitude modes almost always tend to have low frequencies. This can be explained since the modes having high amplitudes are often associated with large scale flow structures which generally oscillate at lower frequencies in such flow regimes. 


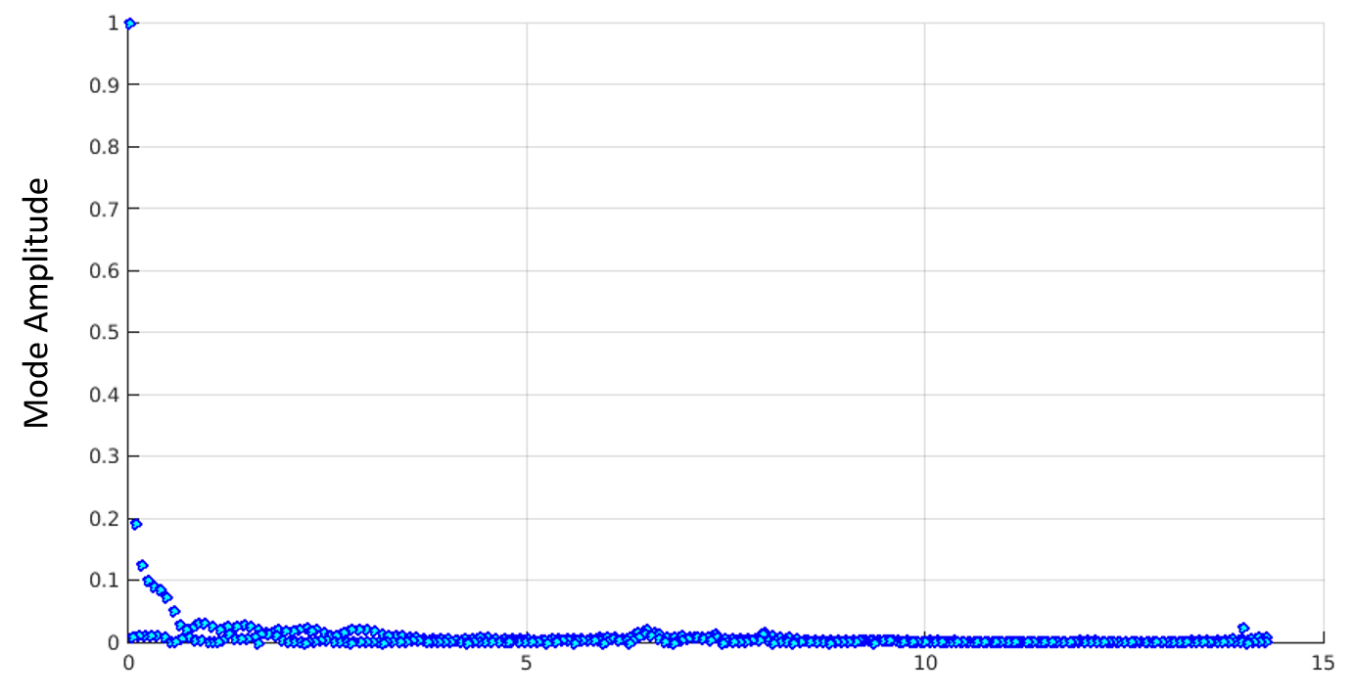

St. No.

Figure 6: Variation of DMD amplitude with frequency for all DMD modes

The spatial structure of the first harmonic mode 5 in Fig. $5 \mathrm{~b}$ shows the incipient shear layer growing from the leading edge (at approximately $\theta=63^{\circ}$, see Fig. 2) during the plunging phase of the airfoil. The flow at this stage is almost fully separated, but the wake shedding has not yet commenced. The wake shedding phenomena and the formation of a trailing edge vortex can be seen at various stages in the $2^{\text {nd }}, 3^{\text {rd }}$ and $4^{\text {th }}$ harmonics in Figs. $5 \mathrm{c}-5 \mathrm{e}$. In particular, the $2^{\text {nd }}$ harmonic in Fig. 5c shows a spatial structure indicating the incipient formation of the LEV. Subsequently, higher harmonics modes 9 and 11 show shedding of smaller wakes - with mode 11 even showing a wake shed from the trailing edge vortex. The modes can be loosely correlated to the observations of Fig. 2 where large wake shedding from the LEV occurs at $\theta=127^{\circ}$, followed by shedding of several smaller structures at $\theta=170^{\circ}$. These events occur towards the end of the downward motion of the airfoil and it is here that the trailing edge vortex gains 
sufficient strength and a trailing edge wake is shed, as seen in Fig. 2. Since the modes 5 through 11 are harmonics of the dominant dynamic mode 3, the spatial structures shown by these modes may be thought to be a decomposition of a single flow structure which is highly dependent on the airfoil oscillation to undergo flow separation and eventual vortex shedding. Therefore, the dominant dynamic mode and its harmonics are seen to effectively describe the basic physics of the complex flow mechanism of deep dynamic stall. Hence, it would be very beneficial to use these modes for analysis to gain further insight into the problem and reduced order modelling of dynamic stall.

\subsection{POD Modes}

A parallel assessment of the POD modes using the same snapshots is now presented. The discussion of initiation and propagation of the LEV in dynamic stall from Fig. 2 indicates that large scale vortices dominate the behavior of the flow for a significant part of the plunge cycle. To determine the nature of modes with high energy content, we use POD, which orders modes in an optimal manner without ambiguity. The dominant mode 1 is always be the mean flow, shown in Fig. $3 \mathrm{a}$ and the next several modes are shown in Fig. 7. The POD modes show the spatial structures of the high energy flow features. Both the POD and DMD modes were weighted with the the grid cell area as mentioned in Ref [36] and it was seen that the grid weighting did not significantly affect the mode spatial structure and properties compared to the non-weighted results. This is most likely due to the fact that the mesh used in this case is extremely smooth with minimal skewness. Therefore, all results presented in this work are from DMD and POD modes which have not been weighted. Mode 2 in Fig. 7a shows a flow structure with a large recirculation vortex over the chord length, not unlike that observed 
in DMD mode 3: this connection will be investigated in the subsequent section. POD mode 3 in Fig. 7b shows a flow feature with spatial support in both the trailing edge vortex and the LEV regions, in addition to a small positive circulation close to the airfoil surface. The lack of a spatial support in the leading edge area points to the possibility that this flow structure may be associated with incipient wake shedding. POD mode 4 in Fig. 7c highlights a small vortex ejected from the separated flow over the airfoil surface. Further, the flow over the airfoil chord is seen to have a velocity close to the free stream. Similarly, POD mode 5 contains a relatively weak structure emanating from the leading edge. Overall, POD provides a comprehensive picture of the most energetic structures in the flow field. However, from an application viewpoint one often needs to know the dynamics of these energetic structures during the course of stall. For example, POD mode 4 highlights the trailing structure without tracking the dynamic events occurring near the leading edge. A cursory examination of the flow structures in Fig. 2 shows that although a clear trailing edge wake manifests itself from $\theta=143^{\circ}$ to $170^{\circ}$, the flow is extremely active near the leading edge. The absence of leading edge features in POD mode 4 is because the POD algorithm selectively 'picks' only energetic regions of flow structures in their modes, which is consistent with their optimalenergy based formulation. Therefore, the transient behavior of the individual flow frequencies cannot be easily gauged by solely using the POD modes, since each of these modes contain energetic features from multiple flow frequencies. In the next section, we relate the POD and DMD modes by analyzing the frequency content of each POD mode. 


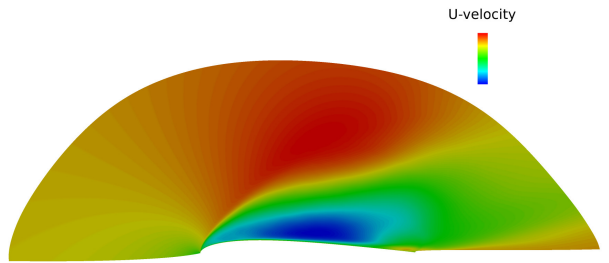

(a) Dominant Energetic Mode 2

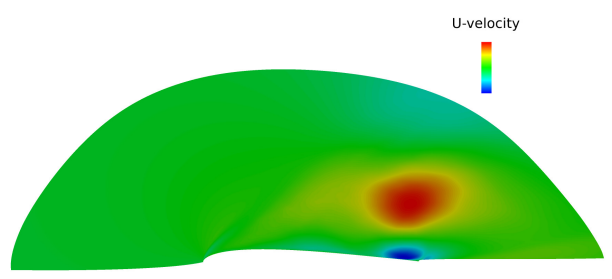

(c) Dominant Energetic Mode 4

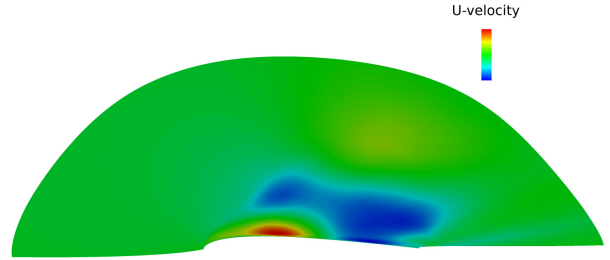

(b) Dominant Energetic Mode 3

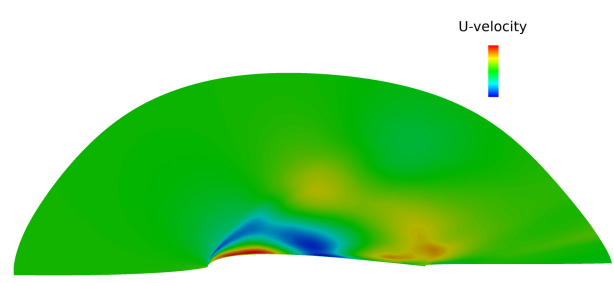

(d) Dominant Energetic Mode 5

Figure 7: Dominant Energetic POD modes

\subsection{Flow Structure Identification from DMD and POD}

The above discussion illustrates the manner in which DMD and POD decompose the LEV and the wake of the plunging airfoil. Here we connect the two types of modes to highlight their complementary nature. A spectral analysis of the POD mode time coefficients can identify different frequencies present in that mode, but not the spatial structure of that frequency or its stability characteristics. On the other hand, DMD isolates flow structures at unique frequencies based purely on their dynamic properties but without considering their energy content. A successful connection enables us to identify highly energetic flow structures along with their transient dynamics, frequency and stability. In other words, using a combination of the two different tools gives better insight into the physics of the flow which could not have been provided by either tool alone.

We first focus our attention on the time varying behavior of the dominant POD 
modes. The evolution of each of the dominant 5 POD modes with time can be computed, since the snapshot matrix can be represented as a linear combination of the POD modes and its time varying coefficients $a(t)_{P O D}$ as given by Lumley [14] for every mode.

$$
\mathbf{V}_{1}^{N}=\sum_{i=1}^{N} a_{i}(t)_{P O D} \Phi_{i}
$$

The time traces of the individual POD modes given by $a(t)_{P O D}$ is used to plot the time evolution of the dominant POD modes in Fig. 8. The time trace $a(t)_{P O D}$ shows that the POD modes are somewhat noisy, especially for mode 1 which is expected to remain constant with time since it represents the mean flow; and this observation is consistent with those made in Ref.[33]. The corresponding DMD traces $\Re\left(a(t)_{D M D}\right)$ are shown in Fig. 9 for the 5 dominant DMD modes. Since the DMD modes are complex, only the real part of the time trace is displayed for comparison. The difference seen in Fig. 9 is immediately apparent, since the time evolution curves are very smooth. A key observation is that the mode 1, which represents the mean flow, is constant in time, which is consistent with its definition. The primary reason for this difference between POD and DMD modes is that POD modes contain flow structures of multiple frequencies, whereas DMD modes contain flow structures of a single frequency. Indeed, this phenomenon was observed in the POD modes in Section 3.2 and is a consequence of the mathematical formulation of POD. Schmid[19] showed that while the POD modes are orthogonal in space, the DMD modes are orthogonal in time. Based on this knowledge, further analysis is performed using the spectral content of the DMD and POD modes to help identify flow structures that are both energetic and dynamic.

To this end, the time signal of the evolution of the dominant POD and DMD 
modes are used to obtain a frequency spectrum. Figure 10 shows the spectral content of each of the dominant POD modes. The spectral content of POD mean mode 1 in Fig. 10a shows the presence of higher frequencies apart from the zero frequency. These are representative of other flow structures, which collectively produce the mean flow in the POD mode 1, thereby leading to its varying time evolution in Fig. 8. As noted earlier, the structure of POD mode 2 has a strong qualitative resemblance to that in DMD mode 3. To explore this observation, the spectral content of POD mode 2 is shown in Fig. 10b. The spectrum has a few dominant peaks occurring at low frequencies. This is expected since large scale turbulent structures having high energy content have low frequency. A closer look at the spectrum reveals that the peak frequency for POD mode 2 is a flow structure having $S t \approx 0.0793$, followed by two smaller peaks of $S t \approx 0.1584$ and $S t \approx 0.2374$ respectively. It is seen that the constituent frequencies in the POD mode match the frequencies of the dominant DMD modes, shown in Table 1. Thus, POD mode 2 appears primarily governed by the dominant dynamic mode from DMD and its $1^{\text {st }}, 2^{\text {nd }}$ harmonics. The peak frequency in POD mode 2 is equal to that of the dominant dynamic mode, which in turn is equal to the imposed plunging frequency of the airfoil. Even though two harmonics are present in the POD mode, the peak frequency is that associated with the dominant dynamic mode comprised of an LEV separating from the leading edge. It is this LEV structure which is seen in the spatial structure of POD mode 2. Thus, the spectral analysis shows that this LEV dominant frequency is not only the most dynamic, but also the most energetic flow structure in dynamic stall, with $S t=0.0793$. This phenomenon is not restricted to just POD mode 2. The spectral content of POD mode 3 in Fig. 10c reveals a peak frequency component of $S t \approx 0.2381$, which is 


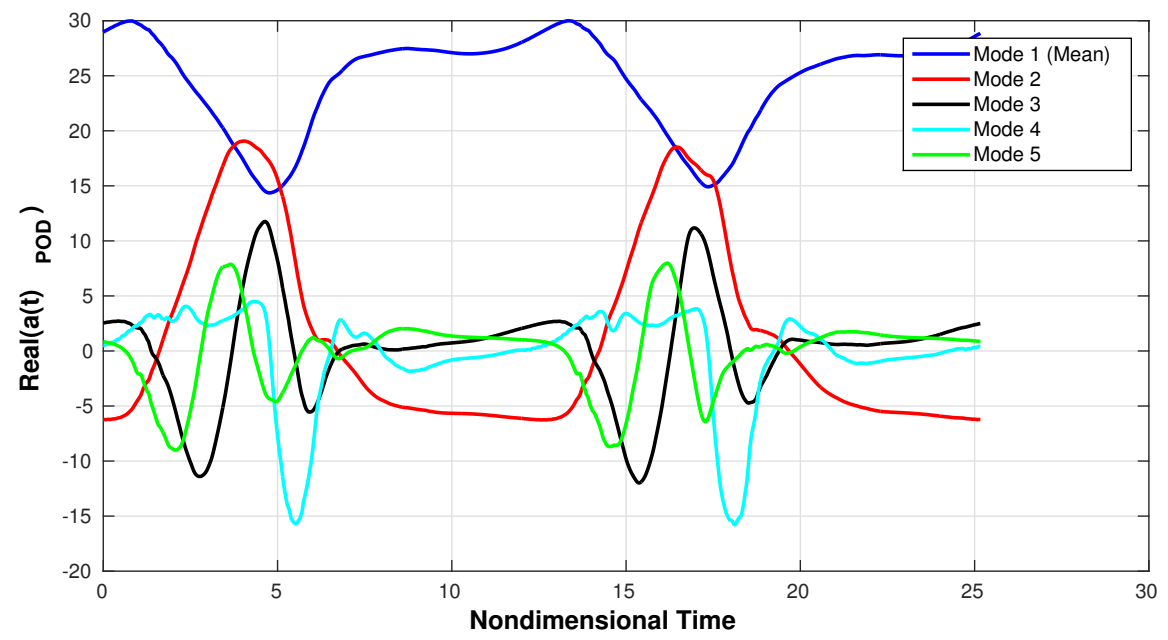

Figure 8: Time Trace of dominant POD modes

very close to $S t \approx 0.2374$ for DMD mode 7 ( $2^{\text {nd }}$ harmonic) with two other peaks corresponding to $S t \approx 0.1584$ and $S t \approx 0.3160$ representing DMD mode $5\left(1^{s t}\right.$ harmonic) and mode $7\left(2^{\text {nd }}\right.$ harmonic $)$ respectively. Thus, the energetic structure in POD mode 3 is governed primarily by the oscillatory dynamics of DMD mode 7 showing a large wake separating when the LEV gets detached. This can also be observed in the POD mode 3 spatial structure showing a large vortex over the airfoil chord.

A similar observation can be made regarding POD mode 4 from Fig. 10d. As discussed previously, the absence of a leading edge flow structure when a trailing edge vortex is present, causes difficulties in identifying the flow structure associated with this mode in Fig. 2. The spectral analysis of this mode shows that several frequencies are present in the flow. The peak frequency of $S t \approx 0.1587$ is associated with the DMD mode 5 ( $1^{\text {st }}$ harmonic). The other components show the presence of flow structures from DMD modes 3, 7, 9 and 11. The flow structure 


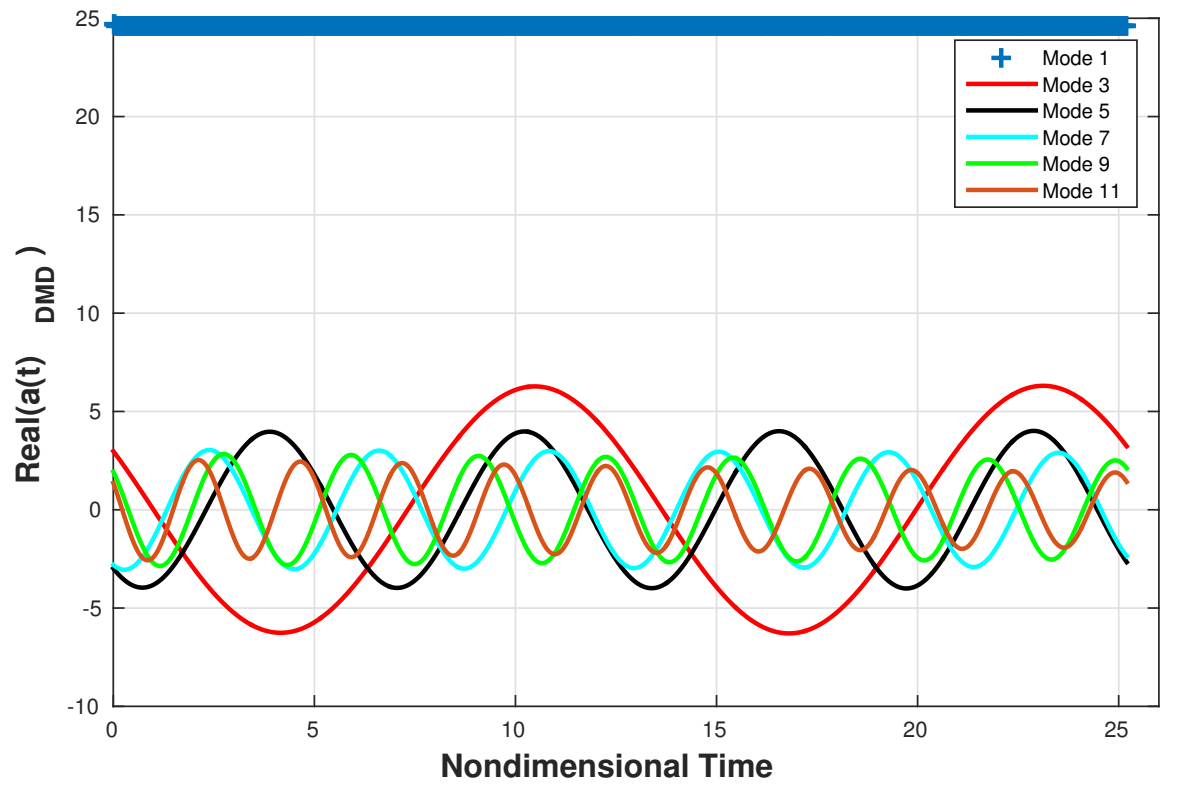

Figure 9: Time Trace of dominant DMD modes

\begin{tabular}{|c|c|c|c|}
\hline POD Mode No. & Peak Frequency & DMD Mode Frequency & Description \\
\hline 1 & 0 & 0 & Stationary Mode (Mode 1) \\
\hline 2 & 0.0793 & 0.0793 & Dominant (Mode 3) \\
\hline 3 & 0.2381 & 0.2374 & $2^{\text {nd }}$ harmonic (Mode 7) \\
\hline 4 & 0.1587 & 0.1584 & $1^{\text {st }}$ harmonic (Mode 5) \\
\hline 5 & 0.3175 & 0.3160 & $3^{\text {rd }}$ harmonic (Mode 9) \\
\hline
\end{tabular}

Table 2: Presence of DMD Mode frequencies in the POD spectrum 

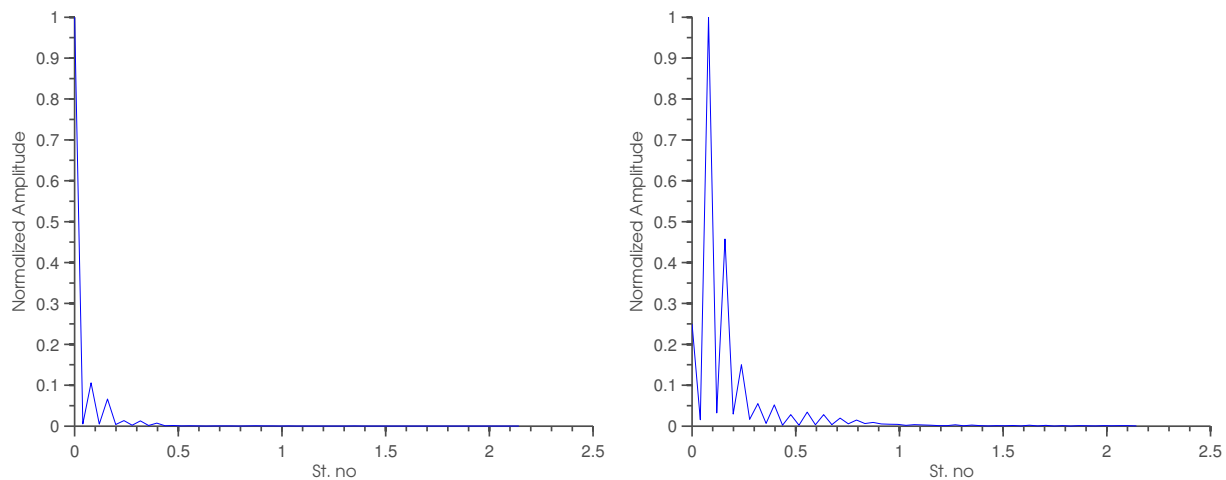

(a) FFT of Mode 1 time signal, Peak $S t=0$ (b) FFT of Mode 2 time signal, Peak $S t \approx$ 0.0793
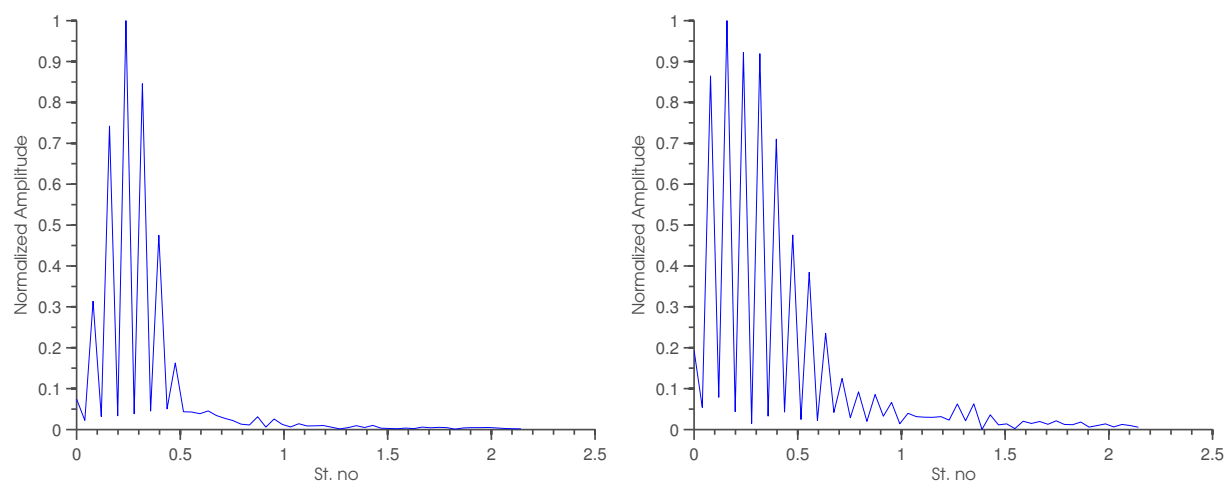

(c) FFT of Mode 3 time signal, Peak $S t \approx(\mathrm{d})$ FFT of Mode 4 time signal, Peak $S t \approx$ 0.2381 0.1587

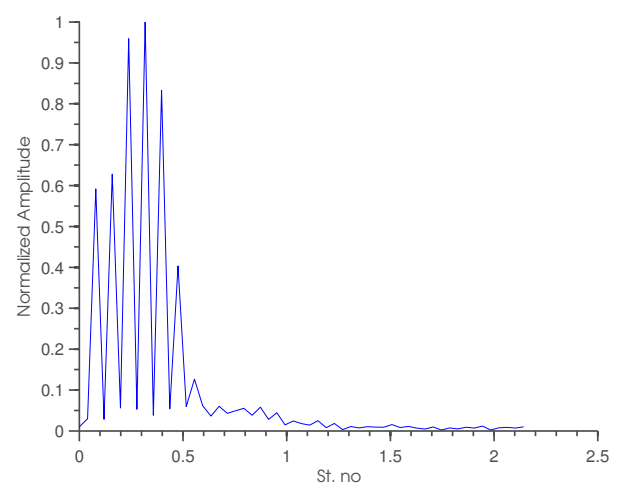

(e) FFT of Mode 5 time signal, Peak $S t \approx$ 0.3175

29

Figure 10: Spectral Content of Energetic POD modes 
in DMD mode 5 in Fig. 5b shows the LEV forming from the leading edge. Since this structure is a first harmonic which has not yet fully formed,it bears some resemblance to the flow at $\theta=63^{\circ}$ during the downward plunge of the airfoil. Finally, the spectral content for POD mode 5 is shown in Fig. 10e. The peak frequency of $S t \approx 0.3175$, representing DMD mode 9 , is present along with frequencies from DMD modes 3, 5, 7 and 11. The DMD mode 9 shows small wakes separating from the recirculating region over the airfoil and the trailing edge and it is this feature that dominates POD mode 5.

Overall, we note that the spatial structure in POD modes 2 and 3 are quite similar to those in DMD modes 3 and 7 respectively, owing to them being the peak frequencies in the spectrum of the POD modes. However, this behavior does not translate to POD modes 4 and 5. For instance, POD mode 4 does not show the presence of a leading edge structure depicted by DMD mode 5. Similarly, POD mode 5 highlights leading edge separation although its principal frequency is similar to DMD mode 9, which shows shedding small wakes and only a faint presence of leading edge shear. This can be explained by the amplitude of the frequencies in the POD spectra. Figures $10 \mathrm{~b}$ and 10c show that the difference between the peak frequency amplitude and the other frequencies is relatively high, indicating that the flow structure associated with that frequency has a higher contribution to the POD mode than the other frequencies. This is the primary reason behind the fact that POD mode 2 looks very similar to DMD mode 3. Applying the same reasoning to POD modes 4 and 5, one can answer the question as to why their spatial structure is very different from their corresponding peak DMD modes. In Fig. 10d the frequencies associated with DMD modes 5, 7 and 9 are of comparable amplitudes and the peak frequency therefore does not have the sole contribution 
to the POD mode. In addition to these dominant DMD modes, there is presence of higher frequencies which correspond to higher DMD modes 13, 15 etc. The spectral content of POD mode 5 in Fig. 10e shows that DMD modes 7, 9 and 11 have comparable amplitudes, which together influence the POD flow structure significantly. Thus, the dominant POD mode consists of a combination of DMD modes, the relative contributions of which decide the similarity with the spatial structure of the POD mode. Thus, the combination of POD and DMD provides insight into flow structures that are energetic as well as transient. In the present case, it is seen that dynamic stall is primarily governed by the rapid changes in the LEV and its harmonics which also happen to possess very high energy.

\subsection{Modal Content in localized regions}

The analysis thus far has focused on identifying flow structures and their frequencies. These have been global i.e. present throughout the domain. From a control standpoint, leading edge control using actuators would directly influence the flow locally. Successful control authority depends on the manner in which these signals are processed by the flow to yield a global response. Here we combine DMD with point probe time-series analysis to connect the global dynamics of the flow to the local dynamics in a given region of interest. Specifically, we relate frequencies active locally near the leading edge to the global frequencies of the LEV which governs the overall behavior of dynamic stall. Thus, we examine how active the 'global' DMD frequencies are near the leading edge region of the airfoil. Although such an analysis can be conducted by further isolating and analyzing critical time periods in the development of the LEV, model reduction

provides a method to obtain estimates with the available, relatively sparser data over the entire cycle. 


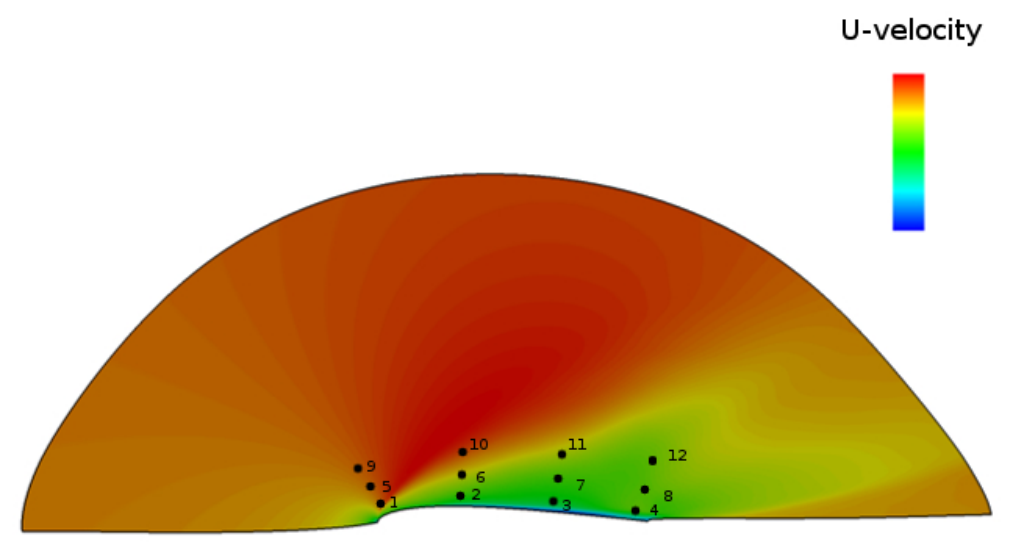

Figure 11: Schematic of Probe locations in flow-field

To carry out this analysis, point probes are introduced in the flow-field at predetermined locations, to obtain the time history of the flow from different regions exhibiting varied physical phenomena. 12 such probes have been chosen, arranged in 3 sets at 4 axial locations, with each set containing probes at different vertical distances as shown in Fig. 11. The distance between subsequent probes in the y-direction is kept constant for all three sets of probes. 1 through 4 lie in the boundary layer region, 5 through 8 in the recirculation region and 9 through 12 closer to the free stream. The same sampling rate has been used throughout this work.

The time signals from the probes are extracted, and FFT analyses are performed. The FFT amplitudes are normalized and compared with the normalized DMD amplitudes. This is a valid comparison since the DMD frequencies are global measures of the frequencies present in the flow field while the probes are the local measures at a given point [13]. Hence the probes capture the frequencies in the global DMD modes but the strength and influence of those modes at any point depends on the physics of the flow at that location. This behavior of the 


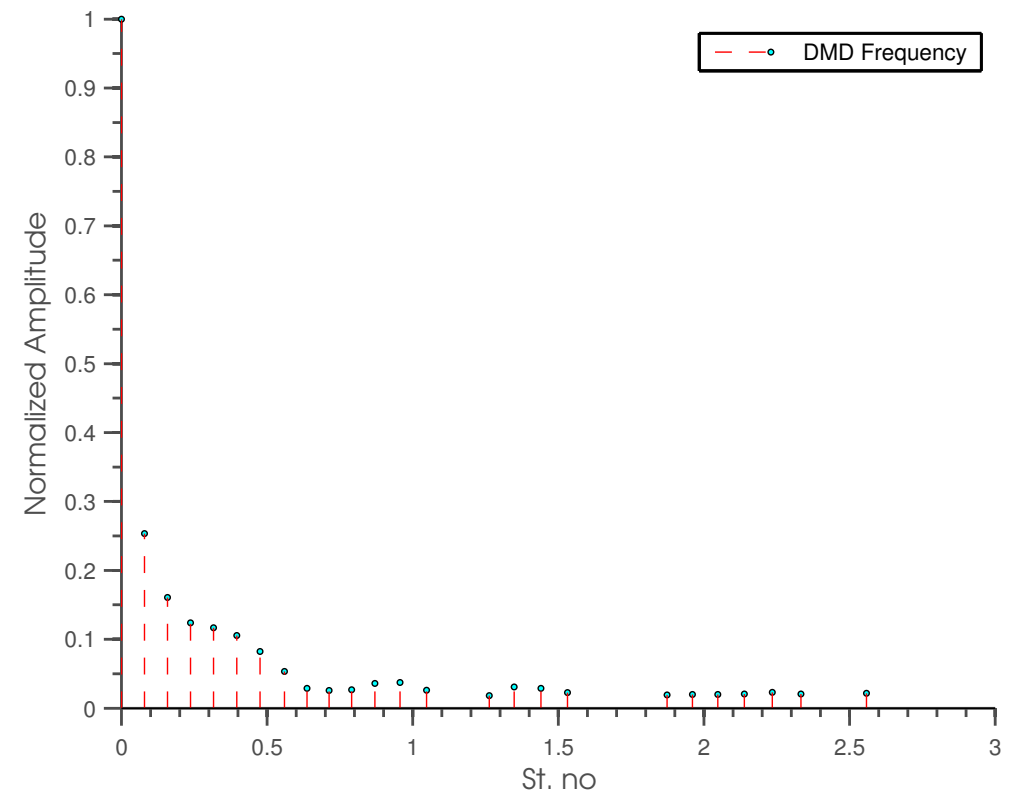

Figure 12: Frequency Spectrum for first 25 DMD modes

global frequencies can be understood by observing the amplitude of the probe frequency spectra. In order to make a valid qualitative comparison, all the amplitudes from the probes and DMD modes are normalized. Since each of the DMD modes has a frequency associated with it based on its complex eigenvalue, the frequency spectrum for the dominant 25 modes (arranged by amplitude) can be plotted, as shown in Fig. 12. The eigenvalues appear as complex conjugates, so the negative frequencies have been discarded so that each mode representing a complex pair has only a positive frequency.

It has been established in literature that the dominant dynamic modes are responsible for most of the critical flow features observed[12, 34]. This trend can also be seen in the present work where the dominant DMD modes are shown to govern the behavior of dynamic stall. Therefore, we consider only the first 15 
DMD modes from Fig. 12 for comparison with the spectral content of the raw time signal from the probes. The comparison has been made to study how the frequencies and their normalized amplitudes from each probe match with DMD spectrum for the entire flow-field. The difference between the two is quantified by computing the $L 2$ norm of the error between the amplitudes of the frequencies from the dominant 15 DMD modes and the amplitudes of the corresponding frequencies from the Fourier spectrum of the probes.

Among all the probes, probe number 12 has the least $L 2$ norm error in amplitude followed by Probe 1 . Probe 9 has the highest $L 2$ norm error. Physically, this means that probes 12 and 1 capture most of the global flow structures (provided by DMD frequencies) using local information (from probes), compared to probe 9. To illustrate this point, consider Fig. 13 where the time signals and spectral content for probes 1 and 9 are shown, superimposed with the DMD spectrum for comparison. Even though Probe 12 has the least $L 2$ norm error, Probe 1 is chosen for study here. This is because Probe 12 lies far above the trailing edge of the airfoil and closer to the freestream. In contrast, Probe 1 is near the leading edge where the shear layer separation occurs which also happens to be the area of interest for flow control.

As discussed previously, the action of an active leading edge actuator is primarily imposed through perturbations at certain frequencies. It would be of immense practical interest to explore how the dominant LEV flow features manifest themselves in the point probes. The dominant mode is the stationary mode with $S t=0$, which is seen in the figure $13 \mathrm{~b}$ superimposed upon the signature of the mean flow on Probe 1. It can be seen that the dominant dynamic mode has a Strouhal number of 0.0793 - which is equal to the plunging frequency, and its 4 


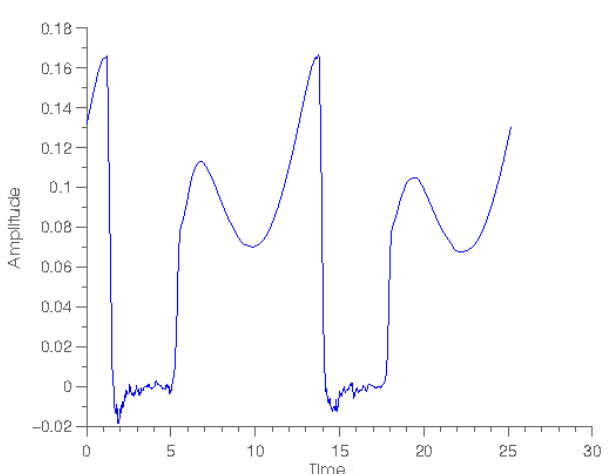

(a) Probe 1 time signal

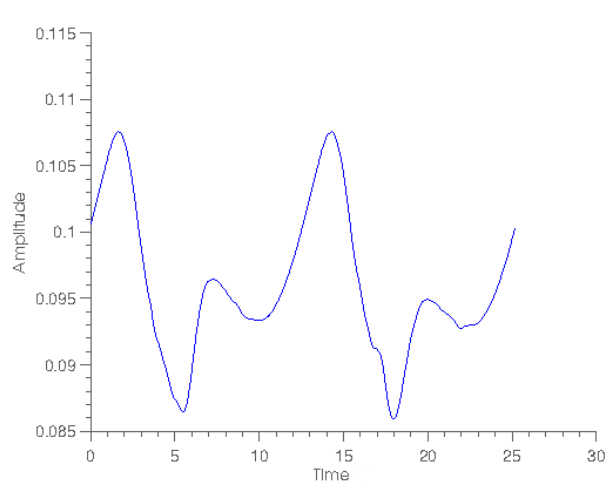

(c) Probe 9 time signal

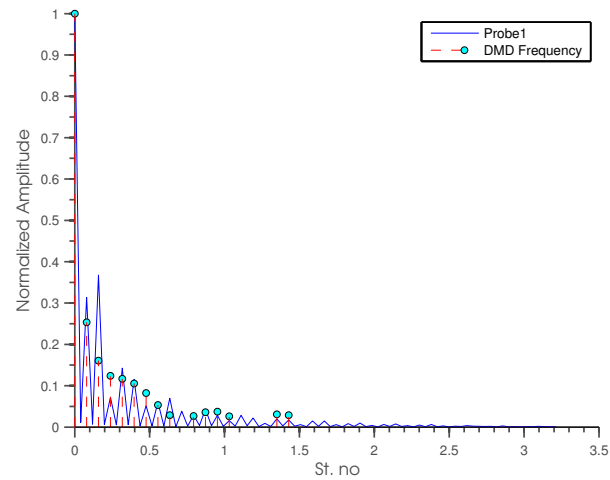

(b) Probe 1 Frequency vs Global Frequency (DMD)

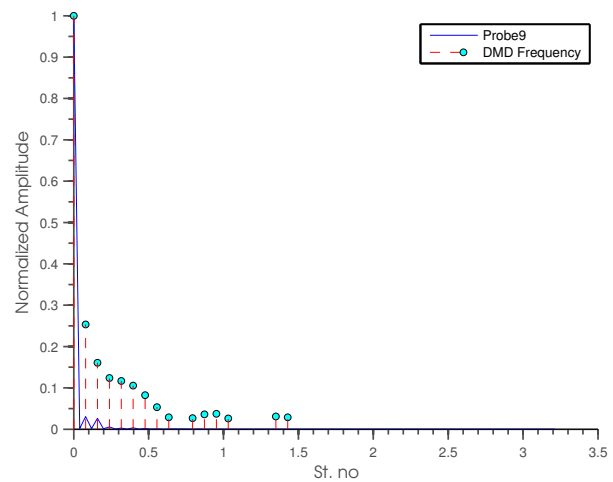

(d) Probe 9 Frequency vs Global Frequency (DMD)

Figure 13: Local Frequency Content of Probe signals vs. Global Frequency spectrum (DMD) 
harmonics are effectively captured. The subsequent modes 7 through 15 are also captured fairly well by the probe, with some deviation in the amplitude.

Figure $13 \mathrm{~d}$ confirms that probe 9 captures only the dominant 3 modes with very poor amplitude resolution and is devoid of any high frequency structures. Since probe 9 position is quite high over the leading edge of the airfoil, it does not "see" the LEV structures during breakdown and thus the information at that location contributes very little to understanding of the mechanism behind flow separation at the leading edge. It appears that the free-stream velocity is the primary influence on probe 9 . In contrast, it is seen that probe 1 in Fig. 13b captures amplitudes of the frequencies very close to that of the global DMD spectrum. This is possibly because the flow separates from the leading edge and the dominant dynamic mode and its harmonics representing the flow originate from that region during the airfoil descent. Since it is only these flow features which govern the primary dynamics of the flow, the data from probe 1 can be very useful for further analysis. Table 3 compares the frequencies of dynamic modes computed from the FFT analyses of the probes locally to the actual frequencies of those DMD modes globally. It is evident that the match is extremely good, especially for the dominant dynamic modes and its harmonics.

Combining DMD with time-series at select points thus yields a good estimate of the strength of the signature of a particular global flow structure at a given point location. Such insight is difficult with time series analyses alone, but in conjunction with DMD, it provides significant information that would have been difficult to obtain independently. Additionally, based on the $L 2$ norm errors from a set of probes we can identify which probe provides the "best representation" of the global flow-field with information obtained from a point in the flow. In essence, 


\begin{tabular}{|c|c|}
\hline FFT Frequency & DMD Mode Frequency \\
\hline 0 & 0 \\
\hline 0.0793 & 0.0793 \\
\hline 0.1587 & 0.1584 \\
\hline 0.2381 & 0.2374 \\
\hline 0.3175 & 0.3160 \\
\hline 0.3968 & 0.3960 \\
\hline
\end{tabular}

Table 3: Comparison of Frequencies from FFT and DMD

this representation provides the best mapping between the local to the global flow and can also be of use in physics-based a priori decisions on the probe locations for experiments and other diagnostics.

\subsection{Reconstruction}

One of the key advantages of model reduction algorithms is the ability to reconstruct the original flow-field using a few important modes which have been found to be relevant from the analysis in the previous sections. Since POD modes are the optimal energy bases for a given dataset, they are very well suited to reconstruct the flow from a minimal number of modes. In this section, we explore the use of DMD modes to perform the same task. A linear combination of individual DMD modes, scaled by their amplitude coefficients, yields the original flow-field[13]. These relative weights (or amplitude) $d$ of every mode are computed using the least squares technique such that the residuals at all time instants and spatial locations are minimized. To begin, each of the dynamic modes can be 
scaled by

$$
\mathbf{v}_{i}=\sum_{k=1}^{N} \lambda_{k}^{i-1} \phi_{k}, \quad i=1, \ldots, N
$$

where $\phi$ are the modes scaled by their amplitudes. At the first snapshot, $\lambda_{k}=1$ since $i=1$ and the equation reduces to

$$
\mathbf{v}_{1}=\sum_{k=1}^{N} d_{k} \hat{\phi}_{k}
$$

where $\hat{\phi}_{k}$ are the unscaled modes and $d_{k}$ are the scaling amplitudes. This can be expressed in matrix form as

$$
\hat{\Phi} \mathbf{d}=\mathbf{v}_{1}
$$

and solved using a standard least squares solver. The modes are then scaled with their corresponding $d_{k}$ and the flow can be reconstructed using a pre-determined number of modes using Eq.27, in the form of a Vandermonde matrix [13]. The evolution of each of these modes can be examined by using the appropriate scaling $d_{k}$ and $\lambda_{k}$, to give a complex time signal $a(t)_{D M D}$ - where $a(t)_{D M D}$ is different from the vector ' $a$ ' used previously for the DMD companion matrix in section 2.3.

Figure 14 shows the normalized amplitude of each of the DMD modes, ranked in decreasing order. Since the dominant modes are the only features to be considered, only the first 50 modes are shown. Using this plot, a suitable cut-off value can be chosen to reconstruct the flow with the requisite number of modes. In this case, it is seen that the dominant dynamic mode along with its 4 harmonics have significant contribution to the flow-field, in terms of both its dynamical behaviour and energy content. Hence, a flow reconstruction using these modes along with the stationary DMD mode would provide a good representation of the flow field compared to the original flow field, on a much more tractable scale. Although a 


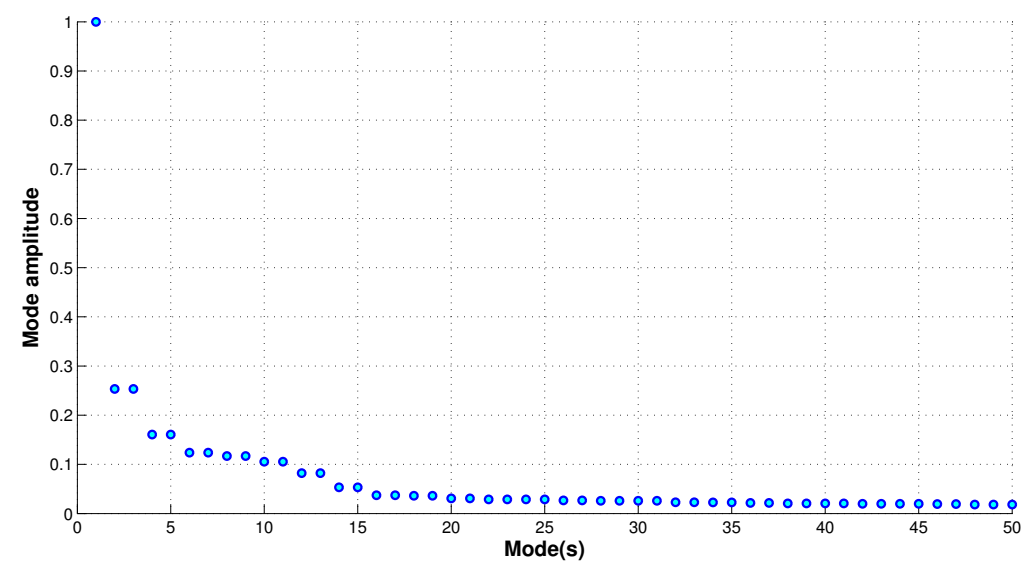

Figure 14: First 50 DMD modes ordered by Amplitude

POD reconstruction is more accurate since it has an optimal representation of the basis for a given dataset, a DMD reconstruction can be used to pick more relevant transient features in complex flow fields such as the one investigated in this work.

For reconstruction, we choose the first 11 modes since they comprise the stationary mode, dominant dynamic mode, their harmonics and their complex conjugates. To compare the extent to which the reconstructed flow matches with the original flow-field, a simple measure of the total error for the reconstruction can be computed as given by equation 30

$$
\text { Error } \%=\left|\frac{\sqrt{\left[\sum_{i=1}^{G}\left(u_{i}^{\text {actual }}-u_{i}^{\text {reconstructed }}\right)^{2}\right]}}{\sqrt{\left[\sum_{i=1}^{G} u_{i}^{\text {actual }}\right]^{2}}}\right| \times 100
$$

where $G$ is the number of grid points. Essentially, Eqn. 30 sums the error throughout the spatial domain across all the snapshots. Using this equation, the resulting total reconstruction error for the flow compared to the original dataset is only $14.53 \%$. To put this in perspective, using roughly just $1.5 \%$ of the 719 modes gives a flow field with $85.47 \%$ accuracy. 
It should be pointed out that caution must be exercised while using Eqn. 30 for all flow cases, since summing errors across time and space will only indicate the total error and will not give information about localized high error regions in the flow. Furthermore, a very high error in a single snapshot can quickly accumulate to give non-physically high total error values. It is thus necessary to compare error percentage for individual snapshots and plot them as shown in Fig. 15. This figure shows the percentage error for every reconstructed flow snapshot for the 11 mode reconstruction of the dynamic stall dataset. The error displays a periodic pattern, following the two periodic cycles of the airfoil trajectory. The error is high in regions where numerous small scale oscillations are present in the flow, especially during wake shedding. However, using just the dominant dynamic mode and its harmonics, the errors are acceptable since the objective is to monitor large-scale behavior as stated in the introduction. The flow is approximated very well towards the end and the beginning of the cycle where the flow is close to being attached. It is seen that all the errors are within the same order of magnitude with a mean error of $13.05 \%$ and a standard deviation of $7.36 \%$. This justifies the use of Eqn. 30 to satisfactorily obtain an overall measure of reconstruction accuracy. In summary, a general rule of thumb to use Eqn. 30 would be to have snapshots with an even error distribution across all snapshots at the same order of magnitude with an acceptable upper bound for the standard deviation.

To compare the reconstruction accuracy near the leading edge, the signals at probe 1 from the original flow field and the reconstructed flow field are plotted in Fig. 16. Since probe 1 lies closest to the LEV separation region and onset of dynamic stall, understanding the transient behaviour of different modes at that location provides insight into formulating a control approach. It can be seen that the 


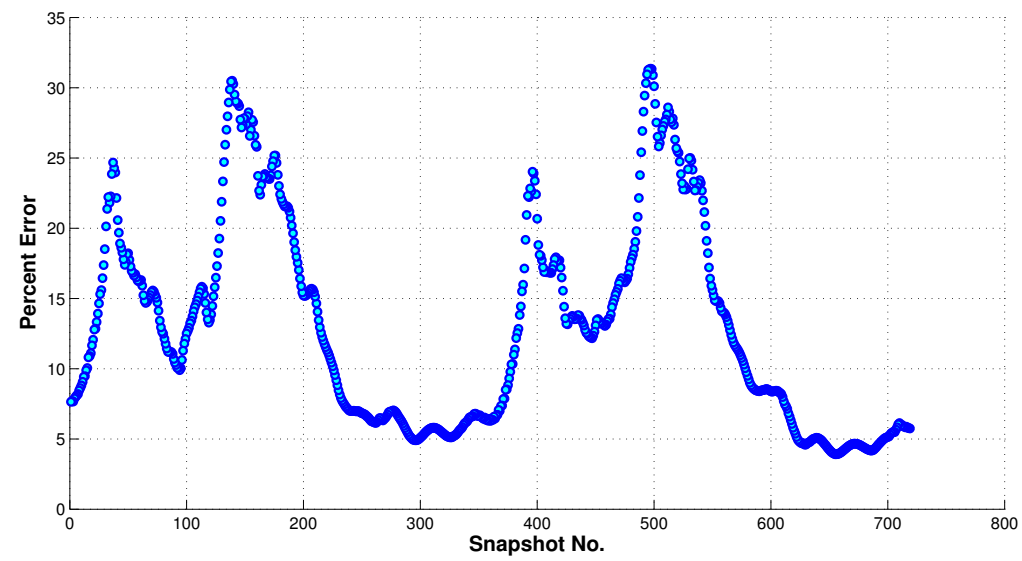

Figure 15: Reconstruction error for every snapshot using 11 modes

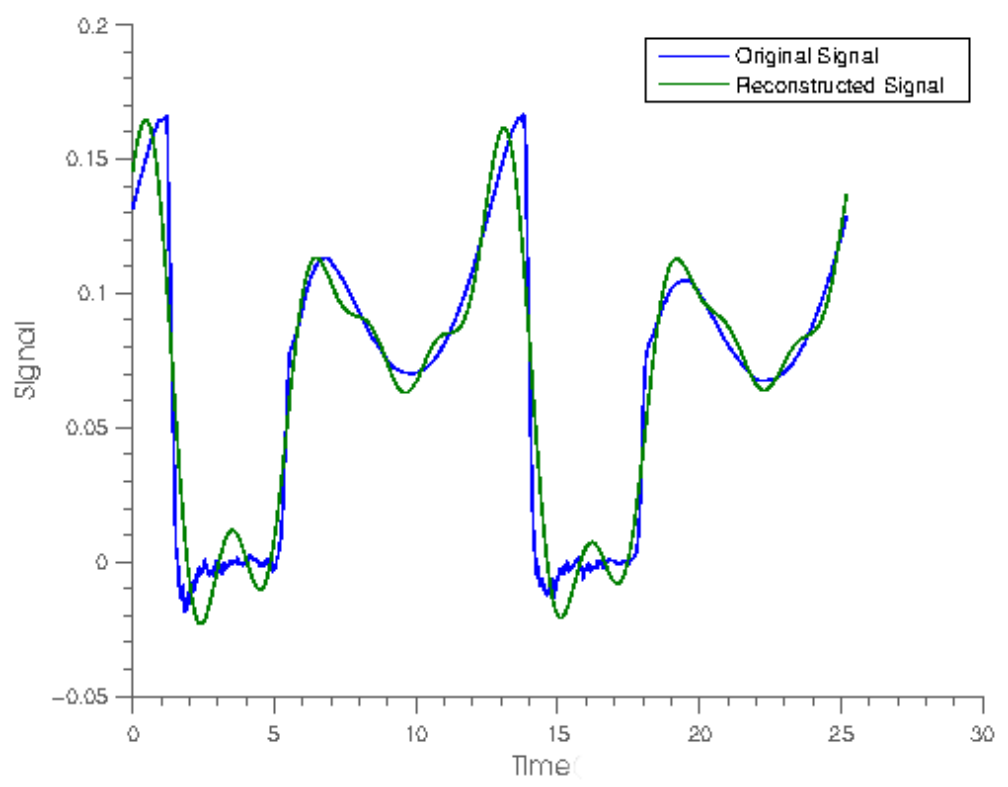

Figure 16: Original Signal vs. Reconstructed Signal at Probe 1 


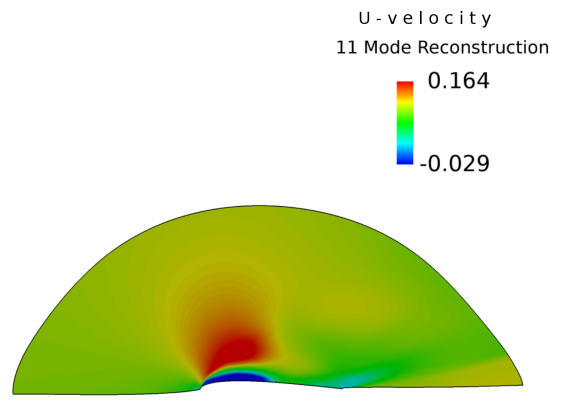

(a) DMD Reconstructed Flow field with dominant 11 modes

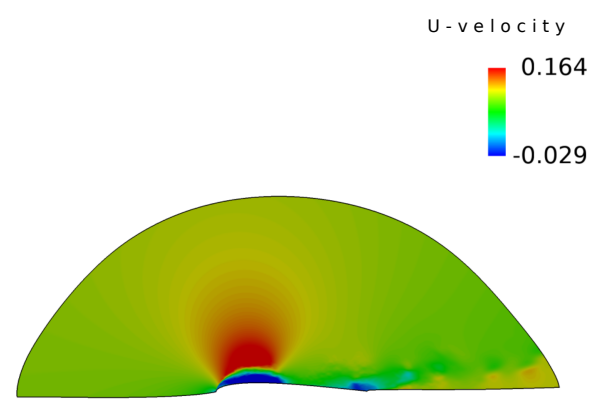

(b) Original Flow field

Figure 17: DMD Reconstructed Flow field vs Original Flow field

dominant mode and its harmonics closely reconstruct the original signal, except in the noisy region between 2 to 5 and 14 through 18 non-dimensional time units. This is most likely associated with the breakdown of the LEV generating numerous extremely small, high-frequency structures which are not captured by the low frequency dominant 11 DMD modes, as also evidenced by Fig. 15. Adding more modes to the reconstruction will address the deficiency in the high-frequency region. However, since the current interest is only in the effect of the dominant dynamic mode and its harmonics, the key features of the profile are captured with good accuracy with an RMS error of 0.0134 relative to the original signal.

Figure 17 shows the reconstructed flow field $17 \mathrm{a}$ and the original flow field $17 \mathrm{~b}$ at the same time instant during the plunging phase of the airfoil. As seen from Fig. $17 \mathrm{~b}$, this is the period when the incipient separation of the boundary layer occurs, before the formation of the LEV. The reconstructed flow consists of very similar flow features compared to the original flow field, with minor differences in the trailing wake region, which the 11 mode reconstruction does not capture 
very well. This is likely due to the fact that only the most dominant modes in the flow-field have been used for reconstruction, whereas using some higher, more dynamic modes can resolve the trailing wake as well. However, for purposes of planning flow control with leading edge actuators, the wake region may be of lesser interest.

\subsection{Flow Structure Identification across Different Velocity Components}

The analysis so far has focused upon identification of flow structures and frequencies using the U-velocity component of the flow. A dominant dynamic mode and its harmonics have been found to govern the dynamics of stall, as seen from the previous analyses and the flow reconstruction in Section 3.5. Since these flow frequencies are a part of a flow structure present in the total velocity field, it would be beneficial to identify parts of this flow structure in the V-velocity component as well. A practical framework for finding these structures using DMD is now described. In order for the flow structures from $\mathrm{V}$-velocity to be considered as a part of the total velocity flow structure, they must have the same frequency as that of U-velocity component for that structure since

$$
U_{\text {total }}=\sqrt{U^{2}+V^{2}}
$$

i.e. a flow structure at any frequency consists of $U$ and $V$ components oscillating at the same frequency. The dominant DMD modes in the V-velocity snapshots are shown in Fig. 18. Mode 3 shows a recirculation region similar to that in U-velocity mode 3. The higher modes show the wake shedding at different stages, and the connection with the corresponding U-velocity modes is not immediately apparent. To further understand this, a search is performed among all the V-velocity modes 
for flow frequencies (imaginary part of the eigenvalue) matching each of the Uvelocity modes. The eigenvalues of the $\mathrm{U}$ and $\mathrm{V}$ velocity modes are ranked by amplitude, and are further filtered by removing the eigenvalues associated with a negative frequency (complex conjugates). The objective is to find V-velocity modes which match the frequency for a given U-velocity mode, within a specified tolerance for deviation. In this work, we specify the deviation of the V-velocity frequency from that of the $\mathrm{U}$-velocity for a given mode not be more than $2 \%$ of the U-velocity frequency.

With this criterion, the V-frequency closest to the U-frequency and also satisfying the deviation tolerance is selected. Applying this methodology for this case, the U-velocity has 360 distinct frequencies. Equivalent V-velocity frequencies were found for 358 of those values. An interesting finding made was that the dominant DMD modes obtained from V-velocity showed the same characteristics as that of the $U$-velocity. Table 4 shows the dominant mode frequencies of the $U$ and V velocity components. It is evident that they match extremely well. Hence, the corresponding modes identify different components of the same total velocity flow structure. Furthermore, the total velocity flowfield can be reconstructed from the dominant dynamic mode and its harmonics for both $\mathrm{U}$ and $\mathrm{V}$ components using the formula in Section 2.3.

$$
U_{i}^{\text {Total }}=\sqrt{\left(\sum_{k=1}^{M} \lambda_{k U}^{i-1} d_{k U} \hat{\phi}_{k U}\right)^{2}+\left(\sum_{k=1}^{M} \lambda_{k V}^{i-1} d_{k V} \hat{\phi}_{k V}\right)^{2}}
$$

for every snapshot. The reconstructed flow using the dominant 11 modes from $\mathrm{U}$ and $\mathrm{V}$ velocities has an accuracy of close to $87 \%$ in comparison to the total velocity original flow-field, which is slightly higher than reconstruction using just the U-velocity. Thus, the flow structure identification has been shown to be successful 


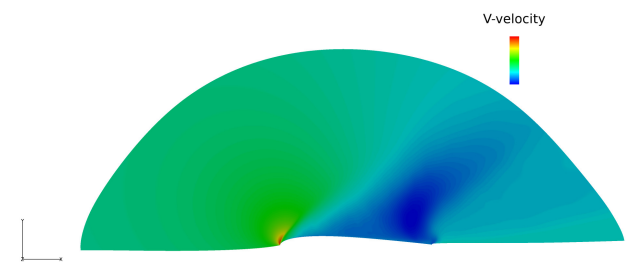

(a) Mode 1 (Mean)

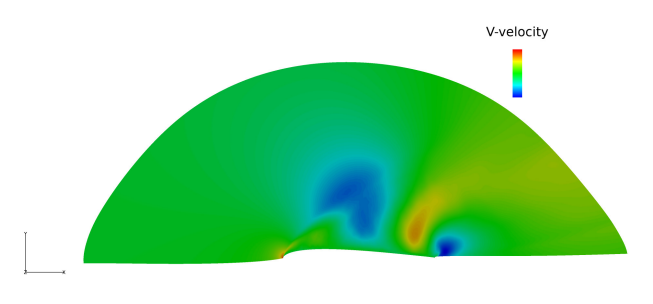

(c) $1^{\text {st }}$ Harmonic Mode 5

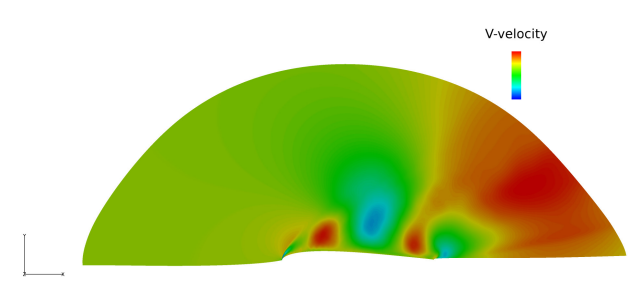

(e) $3^{r d}$ Harmonic Mode 9

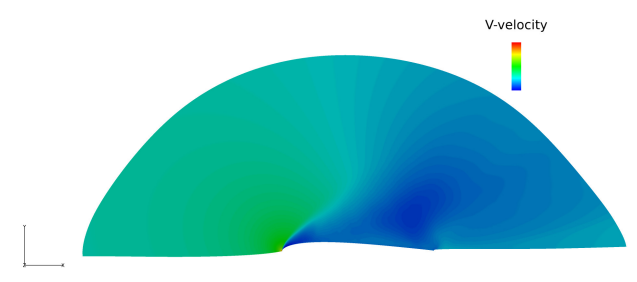

(b) Dominant Dynamic Mode 3

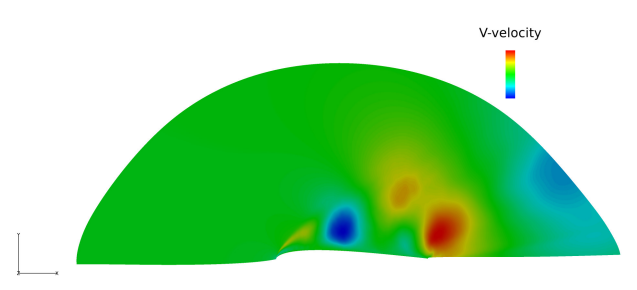

(d) $2^{\text {nd }}$ Harmonic Mode 7

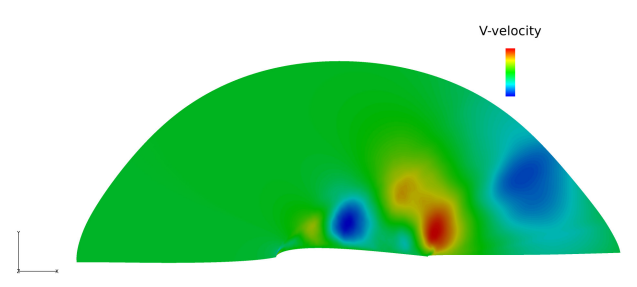

(f) $4^{\text {th }}$ Harmonic Mode 11

Figure 18: Dominant Dynamic DMD modes: V-velocity 


\begin{tabular}{|c|c|c|}
\hline Mode No. & U-Frequency & V-Frequency \\
\hline 1 & 0 & 0 \\
\hline 3 & 0.0793 & 0.0794 \\
\hline 5 & 0.1584 & 0.1588 \\
\hline 7 & 0.2374 & 0.2379 \\
\hline 9 & 0.3160 & 0.3168 \\
\hline 11 & 0.3960 & 0.3940 \\
\hline
\end{tabular}

Table 4: Frequencies of dominant DMD modes

using this technique.

\subsection{Stability Characteristics}

In this section, we use the dominant DMD modes obtained from the U-velocity to examine their stability, as determined from the real part of the eigenvalue. Positive and negative real values indicate growing or damped modes respectively. Thus, the real and the imaginary parts of the eigenvalues can be plotted against each other to obtain an overview of the stability characteristics of all the DMD modes in the analysis. Figure 19 shows the stability characteristics of all DMD modes associated with the airfoil in deep dynamic stall. Several highly unstable modes are present as are a significant number of neutrally stable and stable modes. The unstable modes correspond to flow structures that undergo uncontrolled growth with time, and thus lead to flow separation.

However, as seen in the previous sections and in Fig. 17a, the dominant dynamic mode and its harmonics are the flow structures which primarily govern the flow evolution. Thus, it is insightful to investigate the stability characteristics of 


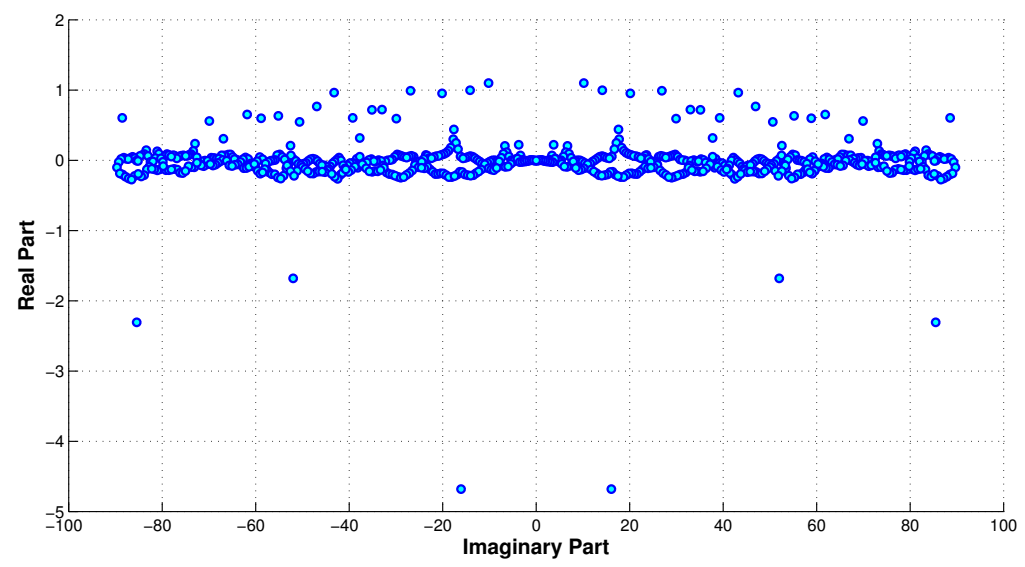

Figure 19: Stability of eigenvalues associated with all DMD modes

these modes and identify the unstable frequencies which may be responsible for dynamic stall. A useful tool to visualize the stability characteristics of such nonlinear dynamical systems is to plot the real and imaginary parts of the mode time evolution (complex) signal as phase portraits.

Figure 20 shows the phase portraits of the dominant dynamic mode 3 and its harmonics. The stability of the DMD modes can be computed using the eigenvalues of the respective modes. Using the stability criterion for eigenvalues mentioned previously, it is seen that modes 3 and 5 are unstable. Modes 7, 9 and 11 are stable. The phase portraits show the degree of stability/instability for a given mode. The dominant dynamic mode 3 is unstable owing to a small positive real eigenvalue, and Fig. 20a shows a trajectory in which the system tends to travel away from the origin very gradually. The direction of the trajectories (clockwise/anti- clockwise) are shown by the arrow in each plot in fig. 20. The first harmonic mode 5 also shows a similar unstable behavior and the separation between the unstable orbits in Fig. 20b is barely visible owing to a very small real part, like mode 3 . 


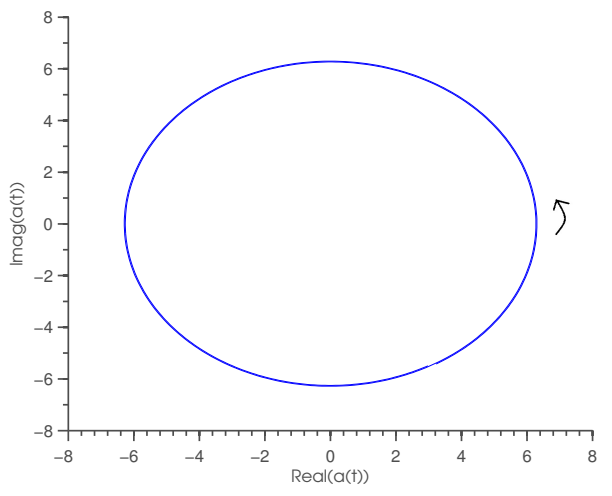

(a) Phase Portrait of Mode 3 time signal

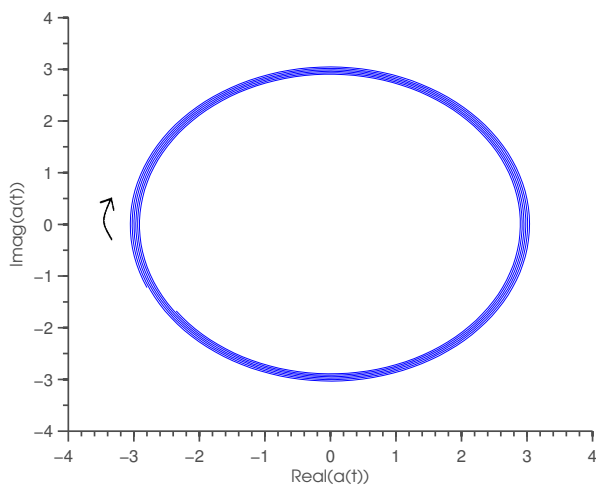

(c) Phase Portrait of Mode 7 time signal

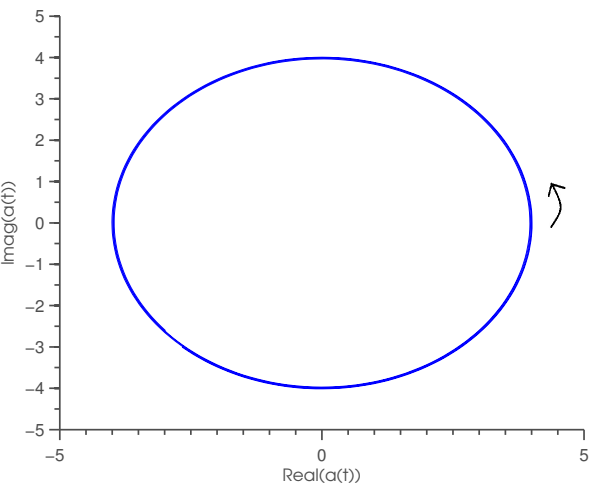

(b) Phase Portrait of Mode 5 time signal

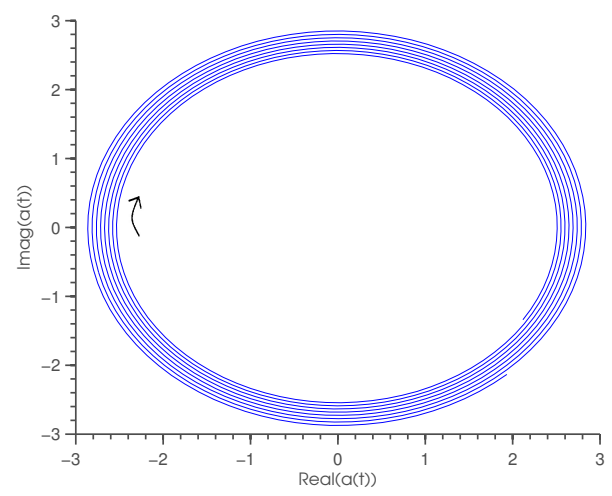

(d) Phase Portrait of Mode 9 time signal

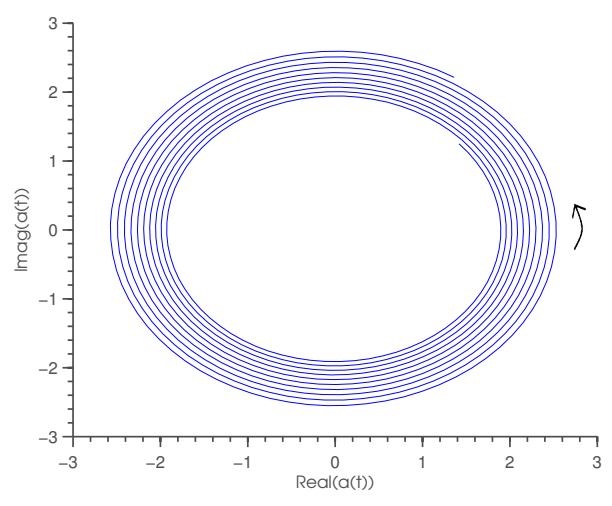

(e) Phase Portrait of Mode 11 time signal

Figure 20: Phase Portraits of dominant DMD modes 


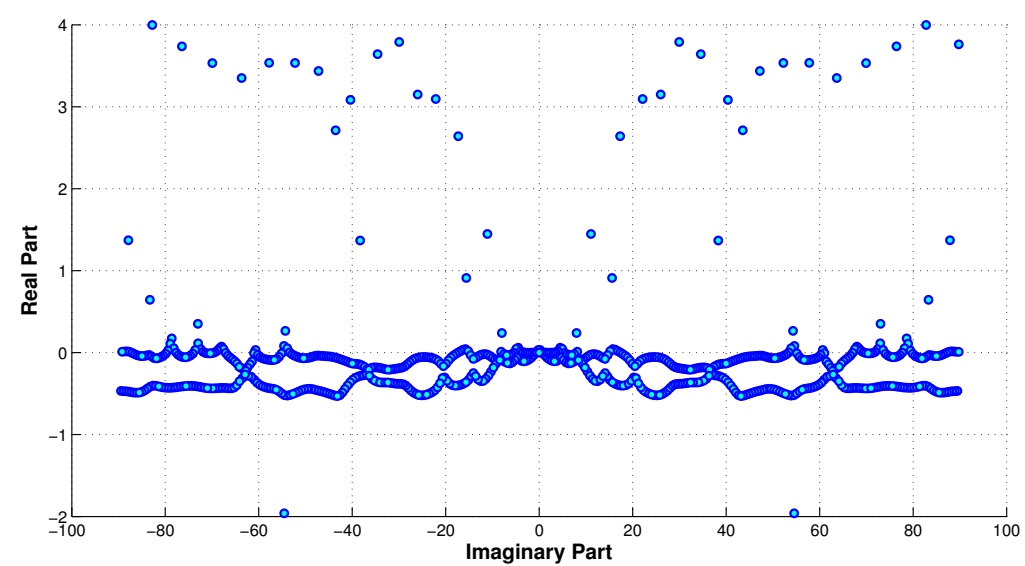

Figure 21: Stability of eigenvalues associated with all DMD modes in the Boundary Layer region

However, the eigenvalue of second harmonic, mode 7 , has a significant negative real part, which is registered in Fig. 20c as an orbit gradually tending towards the origin. Thus, this trajectory is asymptotically stable. Similarly the $3^{\text {rd }}$ harmonic mode 9 is also asymptotically stable, as is the $4^{\text {th }}$ harmonic mode 11 . From the wider gap between the converging orbits, it can be seen that the degree of asymptotic stability is higher for mode 11 compared to mode 9 , which is in turn higher than mode 7 . The stationary DMD mode 1 representing the mean flow is neutrally stable and thus is not shown here.

Similar to the stability analysis performed here for the flow region surrounding the airfoil, another analysis specific to the boundary layer region was performed. The eigenvalues, shown in Fig. 21, show that the boundary layer region consists of highly unstable modes, with magnitudes (Real part) higher than that observed in the full domain analysis, Fig. 19. This indicates the presence of strong instabilities in the viscous layer. An explanation for this is rooted in the phenomena documented in Ref. [11], where small vortices near the trailing edge travel upwards through the boundary layer towards the leading edge, at $\theta=27^{\circ}$ in Fig. 2 . 
These small structures eventually disrupt the laminar shear layer, leading to its breakdown by $\theta=50^{\circ}$.

\section{Conclusion}

The present work uses Dynamic Mode Decomposition to extract insight into dynamic stall in plunging airfoils through analysis of a series of snapshots obtained from LES. The DMD modes identify a dominant dynamic mode consisting of a large recirculation region over the airfoil, oscillating with the same frequency as that of the airfoil itself. Further, analysis of four of the dominant dynamic mode harmonics indicate that the dominant dynamic mode and its first harmonic are unstable. A POD analysis was also performed on the same dataset to identify the most energetic flow structures in the dynamic stall process. A spectral analysis of the dominant POD mode time coefficients shows that each of the dominant POD modes is composed of mainly the dominant dynamic DMD mode and its harmonics. When the peak frequency in a POD mode dominates the others, there is similarity between POD and the DMD mode at that frequency. When the POD spectrum contains multiple frequencies of comparable amplitude to the peak frequency, the spatial structure of the POD mode has no counterpart in the DMD spectrum. POD and DMD have been combined to identify flow structures that have highly transient features relevant to dynamic stall and are also energetic. The flow structures associated with the dominant DMD modes capture the prominent features of dynamic stall so much that a reconstruction of the flow field with only these 11 modes gives a reconstruction accuracy of close to $86 \%$. Measuring reconstruction accuracy solely using total error is shown to have the potential to be misleading, and an alternative is suggested to yield a better physical un- 
derstanding of the propagation of error in the reconstructed flow. Although most results are obtained with the U-velocity, the influence of variable is examined by considering V-velocity as well. The combined results from the two variables can be used to reconstruct the total velocity feature. One of the primary objectives of this work was to identify flow frequencies near the leading edge responsible for dynamic stall to aid control strategy development. In order to do this, in addition to analyzing the flow structures over the entire flow domain the signature of dominant dynamic modes in different regions of interest in the flow have been investigated using point probes. The probe near the leading edge of the airfoil captured local flow frequencies which tracked the global frequencies of the flow structures accurately, better than any of the other probe examined. This indicates that the dominant global modes have a very strong presence near the leading edge region. Finally, a stability analysis of the DMD modes exclusively in the boundary layer region reveals instabilities of very high magnitudes compared to that in the full flow domain, possibly due to upward propagation of disturbances from the trailing edge. Future work would be focused on the shear layer instabilities near the leading edge and mid-chord region to study the key flow components which cause LEV formation. This will be accomplished with data at higher resolutions and sampling rates in these regions of interest to obtain detailed quantitative and qualitative information on the stall initiation mechanism from small instabilities.

\section{Acknowledgements}

This work was supported by the AFRL Collaborative Center for Aeronautical Sciences. The LES of the plunging wing was performed by MV under the sponsorship of AFOSR (Monitor: Dr. Douglas Smith). The simulations were 
carried out using resources provided by the Department of Defense High Performance Computing Modernization Program and Ohio Supercomputer Center. Several figures were made using Fieldview software with licenses obtained from the Intelligent Light University Partnership Program.

[1] W. McCroskey, L. Carr, K. McAlister, Dynamic stall experiments on oscillating airfoils, Aiaa Journal 14 (1) (1976) 57-63.

[2] J. Leishman, T. Beddoes, A semi-empirical model for dynamic stall, Journal of the American Helicopter Society 34 (3) (1989) 3-17.

[3] W. McCroskey, The phenomenon of dynamic stall., Tech. rep., DTIC Document (1981).

[4] L. W. Carr, Progress in analysis and prediction of dynamic stall, Journal of aircraft 25 (1) (1988) 6-17.

[5] M. J. Walsh, Turbulent boundary layer drag reduction using riblets 1.

[6] B. R. McAuliffe, S. A. Sjolander, Active flow control using steady blowing for a low-pressure turbine cascade (2004) 1223-1235.

[7] M. Amitay, D. R. Smith, V. Kibens, D. E. Parekh, A. Glezer, Aerodynamic flow control over an unconventional airfoil using synthetic jet actuators, AIAA journal 39 (3) (2001) 361-370.

[8] M. L. Post, T. C. Corke, Separation control on high angle of attack airfoil using plasma actuators, AIAA journal 42 (11) (2004) 2177-2184. 
[9] D. Roupassov, A. Nikipelov, M. Nudnova, A. Y. Starikovskii, Flow separation control by plasma actuator with nanosecond pulsed-periodic discharge, AIAA journal 47 (1) (2009) 168-185.

[10] C. Rethmel, J. Little, K. Takashima, A. Sinha, I. Adamovich, M. Samimy, Flow separation control using nanosecond pulse driven dbd plasma actuators, International Journal of Flow Control 3 (4) (2011) 213-232.

[11] M. R. Visbal, Numerical investigation of deep dynamic stall of a plunging airfoil, AIAA journal 49 (10) (2011) 2152-2170.

[12] P. J. Schmid, K. E. Meyer, O. Pust, Dynamic mode decomposition and proper orthogonal decomposition of flow in a lid-driven cylindrical cavity, in: 8th International Symposium on Particle Image Velocimetry, 2009, pp. $25-28$.

[13] C. W. Rowley, I. MEZIĆ, S. Bagheri, P. Schlatter, D. S. Henningson, Spectral analysis of nonlinear flows, Journal of Fluid Mechanics 641 (2009) 115127.

[14] G. Berkooz, P. Holmes, J. L. Lumley, The proper orthogonal decomposition in the analysis of turbulent flows, Annual review of fluid mechanics 25 (1) (1993) 539-575.

[15] B. Patte-Rouland, G. Lalizel, J. Moreau, E. Rouland, Flow analysis of an annular jet by particle image velocimetry and proper orthogonal decomposition, Measurement Science and Technology 12 (9) (2001) 1404.

[16] S. Roudnitzky, P. Druault, P. Guibert, Proper orthogonal decomposition of 
in-cylinder engine flow into mean component, coherent structures and random gaussian fluctuations, Journal of Turbulence (7).

[17] L.-H. Feng, J.-J. Wang, C. Pan, Proper orthogonal decomposition analysis of vortex dynamics of a circular cylinder under synthetic jet control, Physics of Fluids (1994-present) 23 (1) (2011) 014106.

[18] K. Mulleners, M. Raffel, The onset of dynamic stall revisited, Experiments in fluids 52 (3) (2012) 779-793.

[19] P. Schmid, L. Li, M. Juniper, O. Pust, Applications of the dynamic mode decomposition, Theoretical and Computational Fluid Dynamics 25 (1-4) (2011) 249-259.

[20] K. K. Chen, J. H. Tu, C. W. Rowley, Variants of dynamic mode decomposition: boundary condition, koopman, and fourier analyses, Journal of nonlinear science 22 (6) (2012) 887-915.

[21] B. A. Belson, J. H. Tu, C. W. Rowley, A parallelized model reduction library, ACM T. Math. Software.

[22] J. H. Tu, C. W. Rowley, An improved algorithm for balanced pod through an analytic treatment of impulse response tails, Journal of Computational Physics 231 (16) (2012) 5317-5333.

[23] M. R. Jovanović, P. J. Schmid, J. W. Nichols, Sparsity-promoting dynamic mode decomposition, Physics of Fluids (1994-present) 26 (2) (2014) 024103. 
[24] A. Wynn, D. Pearson, B. Ganapathisubramani, P. Goulart, Optimal mode decomposition for unsteady flows, Journal of Fluid Mechanics 733 (2013) 473-503.

[25] A. Cammilleri, F. Guéniat, J. Carlier, L. Pastur, E. Mémin, F. Lusseyran, G. Artana, Pod-spectral decomposition for fluid flow analysis and model reduction, Theoretical and Computational Fluid Dynamics 27 (6) (2013) 787 815.

[26] A. Seena, H. J. Sung, Dynamic mode decomposition of turbulent cavity flows for self-sustained oscillations, International Journal of Heat and Fluid Flow 32 (6) (2011) 1098-1110.

[27] S. Bagheri, Koopman-mode decomposition of the cylinder wake, Journal of Fluid Mechanics 726 (2013) 596-623.

[28] T. W. Muld, G. Efraimsson, D. S. Henningson, Flow structures around a high-speed train extracted using proper orthogonal decomposition and dynamic mode decomposition, Computers \& Fluids 57 (2012) 87-97.

[29] A. Cesur, C. Carlsson, A. Feymark, L. Fuchs, J. Revstedt, Analysis of the wake dynamics of stiff and flexible cantilever beams using pod and dmd, Computers \& Fluids 101 (2014) 27-41.

[30] L. Massa, R. Kumar, P. Ravindran, Dynamic mode decomposition analysis of detonation waves, Physics of Fluids (1994-present) 24 (6) (2012) 066101.

[31] O. Semeraro, G. Bellani, F. Lundell, Analysis of time-resolved piv measurements of a confined turbulent jet using pod and koopman modes, Experiments in fluids 53 (5) (2012) 1203-1220. 
[32] P. J. Schmid, D. Violato, F. Scarano, Decomposition of time-resolved tomographic piv, Experiments in fluids 52 (6) (2012) 1567-1579.

[33] E. Alenius, Mode switching in a thick orifice jet, an les and dynamic mode decomposition approach, Computers \& Fluids 90 (2014) 101-112.

[34] S. Mariappan, A. Gardner, K. Richter, M. Raffel, Analysis of dynamic stall using dynamic mode decomposition technique, AIAA Conference.

[35] C. Pan, D. Yu, J. Wang, Dynamical mode decomposition of gurney flap wake flow, Theoretical and Applied Mechanics Letters 1 (1) (2011) 012002.

[36] S. Mariappan, A. Gardner, K. Richter, M. Raffel, Analysis of dynamic stall using dynamic mode decomposition technique, AIAA Journal 52 (11) (2014) 2427-2439. 